Illinois State University

ISU ReD: Research and eData

Theses and Dissertations

3-16-2021

\title{
"They're Issuing Edicts And Proclamations About Your Life, And You're Expected To Follow Those": Living Up To Communicated Parental Expectations About College
}

Anjali Patel

Illinois State University, anjalik.patel23@gmail.com

Follow this and additional works at: https://ir.library.illinoisstate.edu/etd

Part of the Communication Commons

\section{Recommended Citation}

Patel, Anjali, "'They're Issuing Edicts And Proclamations About Your Life, And You're Expected To Follow Those": Living Up To Communicated Parental Expectations About College" (2021). Theses and Dissertations. 1399.

https://ir.library.illinoisstate.edu/etd/1399

This Thesis is brought to you for free and open access by ISU ReD: Research and eData. It has been accepted for inclusion in Theses and Dissertations by an authorized administrator of ISU ReD: Research and eData. For more information, please contact ISUReD@ilstu.edu. 


\title{
“THEY'RE ISSUING EDICTS AND PROCLAMATIONS ABOUT YOUR LIFE, AND YOU'RE EXPECTED TO FOLLOW THOSE”: LIVING UP TO COMMUNICATED PARENTAL EXPECTATIONS ABOUT COLLEGE
}

\begin{abstract}
ANJALI PATEL
113 Pages

As emerging adults transition into adulthood, continued parental involvement and shared messages from parent to child creates a context ripe for research. Unrealistic and demanding messages about expectations from parent to emerging college student have the potential to harm the college students' perceptions of their own college experiences. While research into the positive impacts of parental involvement has exemplified many constructive outcomes for the emerging adult, little research has discussed how parental overinvolvement, overparenting, and control may lead to parental messages about expectations for emerging adulthoods in their college years that may alter the students' college experiences and perceptions of their own academic success and achievement. The purpose of this study was to examine the parental messages about expectations regarding college that emerging adults recall and how their college experiences may be impacted by these messages. Using the memorable messages framework, this study uncovered types of direct and indirect parental messages about expectations and reasons why emerging adults held these messages as salient. In-depth, qualitative interviews were conducted with 14 participants who self-identified as having received (or still receive) messages from their parents about expectations regarding college. The utterances were categorized according to research questions posed. The utterances used by all participants were
\end{abstract}


then analyzed using thematic analysis. Participants recalled three different types of parental messages about expectations, three different response behaviors, and three different outcomes on their collegiate experiences. Implications of the study, limitations, and future research are then discussed.

KEYWORDS: memorable messages; parental expectations; emerging adults; message outcomes 
“THEY'RE ISSUING EDICTS AND PROCLAMATIONS ABOUT YOUR LIFE, AND YOU'RE EXPECTED TO FOLLOW THOSE”: LIVING UP TO COMMUNICATED

PARENTAL EXPECTATIONS ABOUT COLLEGE

ANJALI PATEL

A Thesis Submitted in Partial Fulfillment of the Requirements for the Degree of MASTER OF SCIENCE

School of Communication

ILLINOIS STATE UNIVERSITY 
(C) 2021 Anjali Patel 
“THEY'RE ISSUING EDICTS AND PROCLAMATIONS ABOUT YOUR LIFE, AND YOU'RE EXPECTED TO FOLLOW THOSE”: LIVING UP TO COMMUNICATED

PARENTAL EXPECTATIONS ABOUT COLLEGE

\author{
ANJALI PATEL
}

COMMITTEE MEMBERS:

Aimee Miller-Ott, Chair

Cheri Simonds

Lindsey Thomas 


\section{ACKNOWLEDGMENTS}

To my greatest motivator, Dr. Jones, thank you for guiding me towards this academic journey. You believed in me from the start and invested your time in my success. Thank you for your immensely positive influence. Thank you to the best thesis chair, Dr. Miller-Ott, for continuously offering me abundant guidance and always knowing my next best move. I know it takes so much of your time - I am so grateful. You supported and uplifted me in so many ways. Thank you, Dr. Simonds and Dr. Thomas, for your support and feedback. Dr. Simonds, you have constantly expressed your belief in my capability since my first day in this program. Without you, I would have never felt capable. Dr. Thomas, you have been such a firm source of motivation and fresh outlook. Without you, I would not have realized so many impactful ways of experiencing and studying communication. Mom (Aparna), Dad (Ketan), Kruti, and Kavita, my family, my foundation, thank you all for your support in my academic endeavors; and, for your patience. I could not have made it through all these years of schooling without you, love you all. To my rock, Harshank, I could not have had such faith in myself without your constant love and support (even when you would just nod, smile, and say, "okay honey, that sounds good, you can do this", and "I am so proud of you"). You never let me doubt myself. I love you. Finally, this thesis is for my loving Baa, Dhiren, and Dada. Although you all may no longer physically be here with me, I feel your love and support each day. Baa, I can feel your beautiful hands holding my face in loving adoration. Dhiren, you better be eating a giant piece of cake to celebrate with me. I know you would have been so happy for, and proud of, me. Dada, I will always feel your shining pride in me. I wish you were here to shine that adorable smile and say "congratulations, Anjali”. In all, thank you, to everyone, for helping me achieve all these great things in life. 


\section{CONTENTS}

Page

ACKNOWLEDGMENTS

CONTENTS

CHAPTER I: INTRODUCTION 1

CHAPTER II: REVIEW OF LITERATURE

Parental Involvement in Emerging Adults' Lives 4

$\begin{array}{ll}\text { Parental Expectations \& Violations } & 6\end{array}$

$\begin{array}{ll}\text { Parental Expectations } & 7\end{array}$

$\begin{array}{ll}\text { Cultural Implications } & 9\end{array}$

Parenting Styles and Parental Involvement 13

$\begin{array}{ll}\text { Parenting Styles } & 13\end{array}$

$\begin{array}{ll}\text { Parental Overinvolvement/Overparenting } & 18\end{array}$

$\begin{array}{ll}\text { Parental Control } & 21\end{array}$

$\begin{array}{ll}\text { Memorable Messages } & 24\end{array}$

Parental Expectations and College Student Outcomes 27

$\begin{array}{ll}\text { Motivation } & 27\end{array}$

Mental Health, Depression, and Burnout $\quad 29$

$\begin{array}{ll}\text { Persistence } & 30\end{array}$

Research Questions $\quad 31$

CHAPTER III: METHODS

$\begin{array}{ll}\text { Participants } & 33\end{array}$

$\begin{array}{ll}\text { Procedures } & 34\end{array}$ 
Data Analysis

CHAPTER IV: RESULTS

College Expectations Message Types

Direct and Prescriptive Messages about College

Indirect and Autonomy-Supporting Messages about College

Direct but Autonomy-Supporting Messages about College

Emerging Adult Responses to Parental Messages

$\begin{array}{lr}\text { Compliant and Avoidant Responses } & 50\end{array}$

Autonomous Responses $\quad 54$

Autonomous and Selectively Compliant Responses 56

$\begin{array}{ll}\text { Emerging Adult Outcomes } & 58\end{array}$

Mental Health, Well-Being, and Academic Motivation/Success 59

$\begin{array}{ll}\text { Summative College Experiences } & 69\end{array}$

$\begin{array}{ll}\text { Reflection on Messages } & 72\end{array}$

$\begin{array}{ll}\text { Conclusion } & 76\end{array}$

$\begin{array}{ll}\text { CHAPTER V: DISCUSSION } & 77\end{array}$

$\begin{array}{ll}\text { Summary of Findings } & 77\end{array}$

$\begin{array}{ll}\text { Implications } & 79\end{array}$

$\begin{array}{ll}\text { Messages } & 79\end{array}$

$\begin{array}{lr}\text { Parental Involvement versus Overinvolvement } & 83\end{array}$

$\begin{array}{lr}\text { Outcomes of Parental College Messages } & 84\end{array}$

$\begin{array}{ll}\text { Limitations } & 86\end{array}$

$\begin{array}{ll}\text { Future Directions } & 87\end{array}$ 
$\begin{array}{ll}\text { Conclusion } & 91\end{array}$

$\begin{array}{ll}\text { REFERENCES } & 92\end{array}$

APPENDIX A: RESEARCH BOARD STATEMENT 106

$\begin{array}{ll}\text { APPENDIX B: EMAIL RECRUITMENT } & 107\end{array}$

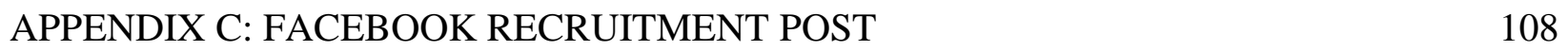

$\begin{array}{ll}\text { APPENDIX D: INFORMED CONSENT } & 109\end{array}$

APPENDIX E: INTERVIEW PROTOCOL 111 


\section{CHAPTER I: INTRODUCTION}

The college years of an emerging adult's life are full of exploration, turbulence, and identity development (Arnett, 2004). In college, emerging adults may make decisions about their lives, including their future careers, that may cause familial turbulence and/or rejection (Harackiewicz et al., 2002). While recent studies have exemplified how parental involvement may serve as a key predictor of students' academic success rates (Barton \& Hirsch, 2016; Silva et al., 2008), others exemplify how parental overinvolvement may hinder autonomy and set up expectations for what the college student's college experience should entail and what decisions should be made (Hesse et al., 2018).

Indeed, today, emerging adulthood looks "more like a stage of life than a swift and coordinated set of transitions" (Furstenberg, 2015, p. 16). Due to the vulnerable nature of such a turbulent and unsure stage of development into adulthood, parents maintain an active role in the emerging adult's life. Here, parents move between providing support through decision making processes and allowing the individual their autonomy to emerge into their own adult life (Faherty et al., 2020). Recent studies have exemplified how this natural tendency has led to parental overinvolvement and overparenting in some cases. For example, helicopter parenting, the excessive involvement of parents in their child's development with the use of inappropriate tactics, strips college students of their ability to establish autonomy and impedes their ability to navigate difficult experiences without parental guidance (Hesse et al., 2018). More importantly, due to the remaining parental authority and influence in college students' lives, prescriptive and demanding parenting styles may set up unrealistic expectations for educational and occupational outcomes, involvement, success, moral beliefs, and behaviors to the detriment of the emerging 
adults' perceptions of their college experiences and academic success and achievement (Agliata \& Renk, 2007; Baumrind, 1991; Faherty et al., 2020).

The potential for unrealistic and demanding expectations from parent to emerging college student to hinder or harm the college students' perceptions of their own college experiences (e.g., academic success and achievement) remains a research area prime for development. While research into the positive impacts of parental involvement has exemplified many constructive outcomes for the emerging adult, little research has discussed how parental overinvolvement, overparenting, and control may lead to the development and communication of parental messages about expectations into emerging adulthood/college years that may alter the students' college experiences and perceptions of their own academic success and achievement.

This study investigates the impacts of parental messages about expectations on college students' perceptions of their college experiences by examining the way college students define their personal college experience realities. Using the memorable messages framework, the goal of this study was to uncover college students' experiences with the messages about expectations that parents communicate to them. In the following chapter, I examined the previous literature on emerging adulthood and parenting styles/involvement before analyzing how parental overinvolvement, overparenting, control, and expectations may impact the college students' perceptions of their own college experiences. 


\section{CHAPTER II: REVIEW OF LITERATURE}

Roughly spanning eighteen to twenty-nine years of age, the emerging adulthood stage of development is characterized as a period for independence, responsibility, personal growth, and exploration (Arnett, 2004; Scheinfeld \& Worley, 2018). Within this extended time period, the emerging adult affirms their existing identity, acquires a new identity, and discovers a desired identity (Karp \& Holmstrom, 1998) while making gains towards self-sufficiency with the ultimate goal of committing to an adult role in society (Faherty et al., 2020).

Perceiving themselves as in control and autonomous, emerging adults undergo the process of individuation wherein individuals navigate different self-identities in their search of their adult role. Here, emerging adults begin to temporarily turn their focus away from some relationships (e.g., parents and siblings) in order to focus on their own self-development (Scheinfeld \& Worley, 2018). Due to the nature of this identity formation process, emerging adults often report feeling in-between, uncertain, and unstable regarding matters including, but not limited to, identities, relationships, and their role in society (Tanner et al., 2016).

As emerging adults attempt to navigate their new autonomy, configure new relationship boundaries with their parents, and understand how the relationship should look, mixed messages create a sense of uncertainty (Scheinfeld \& Worley, 2018). While attempting to develop their autonomy, emerging adults may still need to seek various forms of parental support (e.g., financial). This dynamic tension between autonomy and support may present a certain strain or lack of clarity within the parent-child relationship wherein parents may even begin to perceive themselves to be co-owners of the emerging adults' otherwise personal information (Scheinfeld \& Worley, 2018). During this evolving stage of the emerging adult's transition into adulthood, the parent-child relationship may transform into either a friendship wherein both parties 
understand their pertinent roles (e.g., mother and daughter); in other cases, the parent and child relationship may evolve to become relationally distant or estranged (Scheinfeld \& Worley, 2018). In either case, both parent and child are responsible for the reorganization and reintegration of new, developed relationship identities, roles, responsibilities, and behaviors (Nelson et al., 2007).

Within this review of literature, I first outline the role of parental involvement in emerging adults' lives. I then outline one common source of parental expectations - various parenting styles on childhood and emerging adulthood development. Finally, I detail the overall potential for parental expectations to influence the emerging adults' collegiate experiences and then lead into the research questions of this study. The purpose of this study is to examine messages about college that parents communicate to emerging adults and how their college experiences may be impacted by messages about parental expectations.

\section{Parental Involvement in Emerging Adults' Lives}

Given the ambiguity of emerging adulthood and the remaining need for parental support/guidance, parents often remain active in their children's lives through their 20's (Faherty et al., 2020). However, parents must work to balance providing support and being involved with developing children's autonomy to help the emerging adult develop into their adult identity. It is important to study emerging adults in college, and their relationships with their parents, because the way that they are raised can have important implications for their college experiences. For instance, Dorrance Hall and Scharp (2018) suggest that family communication patterns play a large role in the collegiate students' ability to adapt and remain within an educational institution as an autonomous adult. The higher ability for parents to communicate messages that promote autonomy and allow for self-decision making during this exploratory life stage is imperative 
towards the emerging adults' identity and self-development (Faherty et al., 2020). As children emerge from their high school careers into college, they face a wide variety of differing parenting styles that may impact their development of self-identity (Faherty et al., 2020). It is during this stage of ambivalence and tension where parental ability to communicate autonomy and self-sufficiency while providing requested, yet reserved, support becomes imperative (Dorrance Hall \& Scharp, 2018; Furstenburg, 2015).

The parents' ultimate goal throughout emerging adulthood should be a healthy release into adulthood wherein the child is allowed to obey, think, and feel in an autonomous manner (Furstenburg, 2015, p. 17). Cheung et al. (2019) research the mediating role of positive experiences for emerging adult psychological outcomes. Their results suggest that "savoring" positive family functioning and experiences may serve as a mediator preventative of emerging adulthood acquisition of depression. Here, family communication and experiences that invited autonomy and personal development led to positive perceptions of family functioning. At the collegiate education level, manifestations of parental control, whereby parents try to largely influence emerging adults' journey towards adulthood, may become quite detrimental to the development of the adult (Faherty et al., 2020, p. 697). For instance, emerging adults may feel pressured or obligated, through parental messages, into certain careers, worldviews, identities, and/or relationships. Instead of pursuing and exploring independently, emerging adults with parental control may emerge manifested with feelings of lost identity, insufficiency, and selfdoubt. Overall, developmental environments that are inhibitive of autonomous functioning and decision making (e.g., unrealistic parental expectations) can thwart individuals' self-growth and self-esteem (Ryan \& Deci, 2000). 
Recent research has indicated the evolving presence of parental involvement within the emerging adult transition into adulthood (Nelson et al., 2010; Silva et al., 2008). Additionally, a fair amount of work has been conducted regarding the parent-child collegiate relationship and variables such as living arrangements, economic support, and attachment (Furstenburg, 2015; Nelson et al., 2010; Ryan \& Deci, 2000). However, while much of the work in these areas focuses on the parent-child relationship, specific work on parental messages is much more limited (Nelson et al., 2010). Further, little is known about how parental expectations may influence emerging adults' decisions, development, and their perceptions of their collegiate experiences. At the nexus of parental overinvolvement, control, and expectations are the interactions between parents and their emerging adult children. Therefore, in the following sections I discuss parental expectations and violations alongside a few cultural implications; and finally, I detail the source of parental expectations - various parenting styles and parental involvement.

\section{Parental Expectations and Violations}

Throughout the developmental years, familial interactions may serve as a key predictor of emerging adult mental and physical outcomes. To develop a more robust understanding of parental expectations, this thesis will also provide a review of cross-cultural relational differences and implications on interaction styles/expectations. Miller-Ott \& Kelly (2015) highlight that "in more established relationships, partners may create expectations specific to that person based on prior knowledge of the other, relational history, or observation" (p. 254). This aspect of specificity towards certain relational partners creates an interesting context for study within parent-child relationships. Cionea et al. (2018) study the implications cultural differences 
can have on specific expectations and indicate that expectations can be predicted by one's culture, relational models, sex, and gender roles.

Research suggests that cultural implications, parenting styles, and childhood development may have an impact on the types of messages parents convey to evoke expectations or respond to violated expectations (Cionea et al., 2018). Perhaps the familial relationship creates a less ambiguous context through which violations are construed as positive or negative based on familial bonds and established behavioral or communicative norms. This thesis will seek to understand what types of memorable messages emerging adults recall having received (or who still receive) about parental expectations about college, how emerging adults respond, and how these parental messages influence collegiate students' perceptions of their own collegiate experiences. To further the reader's understanding of expectations, I first outline a few studies that exemplify the nature of parental expectations; then, I discuss some of the cultural implications/differences present within the research.

\section{Parental Expectations}

Parental influence and expectations on collegiate students' adjustment are frequently underestimated (Agliata \& Renk, 2007). Growing research has exemplified that regardless of how unrealistic expectations are perceived by the emerging adult, parents' expectations are of great concern and a great source of stress to the collegiate student (Agliata \& Renk, 2007; Archer \& Lamnin, 1985; Wang \& Heppner, 2002). As such, students who believe they have not fulfilled their parents' expectations report lower self-worth and adjustment (Agliata \& Renk, 2007). As the emerging adult continues to view their parents as authority figures, they continue to work and seek to meet parental expectations while also formulating an unrealistic image of success. Karp et al. (1998) describe the college transition process for college students as a coming of age 
wherein the emerging adult must adapt within and out of their existing and new identities. However, with the growing influence of continued parenting within the emerging adult's life, Youniss and Smollar (1985) state that many collegiate students still view their parents as guiding/authority figures who may set rules or goals for their emerging adults' life outcomes.

Consequentially, parents may knowingly or unknowingly instill unrealistic perceptions of their expectations by stressing the importance of education, success, occupational outcomes, and responsible social behavior to the detriment of the emerging adults' personal development (Agliata \& Renk, 2007). For example, Alford and Harrigan (2019) detail how emergent daughters place a large emphasis of their adulthood identity development on the role expectations their mothers and family members communicate. Chen and Ho (2012) posit that collegiate students try to interpret their parents' values, beliefs, and expectations and internalize them. When emerging adults in college do not believe they are meeting expectations, they experience a host of negative, intense, and unpleasant emotions and outcomes (Agliata \& Renk, 2009; Higgins, 1987). In attempts to relieve the distress of parental expectations, collegiate students may develop the tendency to use others' expectations as a measure of their own success and ultimately come to define themselves by how closely they have met parental expectations rather than their own achievements over time (Higgins et al., 1986). The importance of the parent-college student relationship within the American culture, and the implications of parental expectations on collegiate student self-development, is often discounted resulting in a gap in the literature regarding this relationship (Agliata \& Renk, 2009; Wang \& Heppner 2002; Weidman, 1989). To further exemplify the nature of parental expectations, and to illuminate the gap within American culture research, I detail a few cross-cultural studies on parental expectations and the impacts on collegiate students. 


\section{Cultural Implications}

Cross-culturally, children approach tasks based on the influence of their parents' culturally based beliefs (Jose \& Bellamy, 2012). These findings suggest that taking cultural variance into account, highly imposed expectations (in this study, academic expectations) predict greater self-doubt and self-critical evaluations of self-performance and self-made decisions. More pertinently, these findings suggest that there are highly relevant cross-cultural differences on the construal of parental messages that elicit control, expectations, and/or demand.

Particular to their study, Jose and Bellamy (2012) detail the differences between Asian (Chinese and Japanese) and European (American) students on effort and persistence. Here, cultural values emphasize the importance of learning, practice, and effort towards the development of intelligence. Academic achievement/motivation is construed through determination and persistent effort to acquire knowledge. Failure, within such entity cultures, is viewed as a lack of intelligence. Thus, the heightened value for intelligence within Asian cultures creates elevated expectations and parental control. Within the Asian cultures, parents are revered and viewed as having the most knowledge through experience. Therefore, the knowledge parents hone, and the willpower/persistence for intelligence they impart creates a heightened expectation within Asian parents for the achievements of their children (Jose \& Bellamy, 2012).

Asian parents hold higher expectations than parents of most other cultural groups and therefore higher predictions for self-doubt become relevant for emerging adult children who fall short of such heightened and somewhat unrealistic expectations (Naumann et al., 2012). In their cross-cultural comparison between Asian and Latino collegiate students, researchers found strong implications for educational attainment to be related to ethnic background. Parental expectations are partly determined by parental educational achievement (Naumann et al., 2012). 
Here, due to the nature of Asian individuals' beliefs and values to strive towards higher degrees, Asian parents (over Latino) expect their children to obtain higher educational status and degrees. In turn, Asian students report higher moral and social obligation to achieve and be successful and worry about falling short of expectations (Naumann et al., 2012).

As a form of child behavior manipulation, parents may also enact messages of demand or psychological control. Rudy et al. (2014) studied parental tendencies to manipulate child behavior via messages of demand and psychological control between India and The United States. Highlighted within their study are the differences between perceptions of morality on psychological control and manipulation. Selfless behavior, within the Indian culture, is a key way of living and of high value. According to beliefs in "Dharma," exemplary behavior exists where the self and social order are not opposed and doing one's duty involves fulfilling societal and parental expectations and parental order (Rudy et al., 2014). Within their study, guilting, in the Indian culture, was not significantly related to perceptions of psychological control, but rather, parental interest and right. Therefore, parental guilting may be differently associated and defined with psychological control and self-esteem in India; where, in America, guilt and autonomous functioning capabilities are thought to be negatively opposed (Rudy et al., 2014). At the core of these differences in psychological control perceptions may be cultural differences in the valuing of education as a source of parental expectations.

Manifestations of desire for control may be culturally construed and directed through communicated parental expectations. According to Oishi and Sullivan (2005), more specific expectations from Asian parents than European American parents elicited higher quality of life perceptions in European American students than Asian students. Due to more unrealistic and heightened requirements/expectations for fulfillment, Asian students report higher sentiments of 
stress, obligation, and academic responsibility. Finally, the concepts of "family honor" and "shared social image" precipitate a type of interdependence wherein family members (particularly parents and elders) become responsible and involved in protecting the "family honor" by communicating demands, responsibilities, and behavioral control (Rodriguez et al., 2014, p. 400). Here, manifestations of desire for control becomes culturally construed and directed through communicated parental expectations from parent-child.

Understanding cross-cultural differences in education value provides insight into the various reasonings for parental messages that elicit expectations or control within differing cultures. Vitoroulis et al. (2012), in a study between Cuba, Spain, and Canada, found that Cuban individuals tend to place a higher value on educational attainment and achievement than those of Spain and Canada. Due to the higher-based emphasis, Cuban students maintained higher academic intrinsic motivation and personal expectations for achievement. Parental/social support differences, cross-culturally, present differences in self-esteem, pride, shame, and guilt. Ishii et al. (2017) examine the differences between European American and Japanese students. They report that European American students report feelings of self-esteem and pride while Japanese students report feelings of shame and guilt. Social and parental support provide individuals the opportunity to cope better in the face of adversity and crisis and can lead the individual to feel loved, supported, and cared for. Social and parental support is highly determinant on culture. As such, where European American students reported higher self-esteem and pride due to warm perceptions of social and parental support, Asian students reported higher guilt and shame due to failed attainment of parental and personal expectations and perceptions of social and parental support as less likely to occur within the cultural contexts. As a result of such parental and social support differences, differences in life satisfaction and well-being also occur. 
The overarching research suggests that one way to prevent a decline in college students' self-worth and identity development, and an increase in distress would be to look directly upon communication styles/skills between the parent and child (Spitzberg \& Hurt, 1987). For example, parental expectations can be construed through a variety of communication contexts (e.g., culture and beliefs). These cross-cultural differences in expectancy development, academic achievement/value, quality of life, and family communication, given the general nature of this thesis, become relevant to understand as the implications on college students' perceptions of their collegiate experiences can vary based on culturally based emphasis of expectations and parental control messages/behaviors. As most American institutions are home to a diverse range of individuals, cross-cultural differences and implications for these students' perceptions of their collegiate experiences become relevant to understand.

Through the literature on expectations, parental expectations, and cultural implications, the reader may begin to understand the nature, and cultural differences, of parental expectations. Emerging from the literature reviewed is the importance of the communication that takes place between parent-child, and parent-emerging adult, and how parenting styles and involvement may impart an innate, sometimes detrimental, desire to fulfill parental expectations. Communication between parents and their college students about what is truly expected may promote a more accurate foundation for goals instead of expectations (Agliata \& Renk, 2007). However, communication whereby parents attempt to direct an emerging adult's journey towards adulthood via messages about expectations may become quite detrimental to the development of the adult (Faherty et al., 2020). For this thesis, I looked into the latter situation in which parents espouse expectations. To further develop the reader's understanding of parental expectations, it is important to also consider where these expectations may be sourced and developed within the 
family. In the following section, I discuss various parenting styles, parental involvement forms, communication patterns, and messages that may elicit overwhelming perceptions of parental expectations within the collegiate student.

\section{Parenting Styles and Parental Involvement}

At the collegiate level of adult development, parents must navigate a communicated balance between support/warmth and allowance of freedom/autonomy. Parental involvement at the collegiate developmental years has been found to be both beneficial and detrimental (Faherty et al., 2020). When parenting styles, parental involvement, and communication begin to "tip" towards manifestations of control and demand, messages may begin to become more prescriptive and controlling in nature (Faherty et al., 2020). Here, the emerging adult internalizes messages and begins to have no authority over their family life and experiences. It is important then to understand the different ways that parents communicate and families interact that likely play a role in how parents set expectations for their emerging adults.

\section{Parenting Styles}

Various communicative patterns between parents and children can hinder or strengthen the parent-child relationship (Gridley et al., 2018). In a study by Gridley et al. (2018), parents' communication that was characterized by consistent care, compatible behavior, and open communication correlated with children perceiving their relationship with their parent as functional and affectionate. Conversely, parental language and behavior that is demeaning or dictating in nature can lead to the development of distorted and undesirable perceptions of the interaction and result in a negative child perception of the relationship (Gridley et al., 2018; McKinney \& Kwan, 2018). In families that encourage unrestrained interactions involving a variety of topics, participatory decision making, and the freedom of expression, even when 
entailing different opinions, an open communication environment may be more likely to enhance the overall well-being of individual family members and may also garner improved information processing skills and/or more competent communication behaviors (Schrodt et al., 2008). Thus, when parents encourage and communicate unrestrained interaction and decision making, while withholding expectations, the emerging adult may be more likely to garner well-being, autonomy, and fulfilling experiences.

Indeed, parents' interaction style can help foster a positive self-concept for the child and a positive relationship for both when a child experiences identity turbulence during the collegiate transition (Faherty et al., 2020; Spivey et al., 2018). Aldeis and Afifi (2013) examined emerging adults' recollection of messages from peers, siblings, and parents. They examined the emerging adult's willingness to reveal or conceal behaviors and found that when college students perceived their parents as confirming, they were more trusting and willing to communicate with the parent. Lanza-Kaduce and Webb (1992) found that college students who perceived messages from their parents as controlling were more likely to drop out of school due to poor academic achievement and social maladjustment.

The types of parenting styles that parents adapt during childhood development create the context for family communication and interaction into emerging adulthood (Michalos et al., 2014). According to the American Psychological Association (APA) (2020), the three main types of parenting styles are authoritative (parents may be seen as nurturing yet limiting), permissive (parents are lax and fail to set limits), and uninvolved (parents are unresponsive and rejecting). With authoritative parents, findings support that children may be friendly and self-reliant, while with permissive parents, children tend to be impulsive and aimless (APA, 2020). With uninvolved parents, children will have low self-esteem and low confidence (APA, 2020). 
Baumrind (1971) also indicates four parenting styles that follow along the same continuum: authoritative (high demand and high responsiveness), authoritarian (high demand and low responsiveness), permissive (low demand and high responsiveness), and indifferent or neglectful (low demand and low responsiveness). Parenting styles may be seen as attitudes towards children that shape parenting practices which, in turn, shape parent-child interactions across different settings and situations. Additionally, Leung et al. (1998) and Tang et al. (2018) emphasize that parental style is provisional of the emotional climate for interaction between parent and child and may have a significant impact on quality of life.

Research following the parenting styles framework established has since come to categorize the differing styles into various main categories (e.g., supportive vs. prescriptive, responsive vs. demanding) (Baumrind, 1991; Wolfradt, 2002). Looking specifically into the context of parental expectations, in the following sections, I detail parenting styles that are either supportive or prescriptive and discuss the relationship of these entities to parental expectations for their college student.

Supportive Styles. The authoritative parenting style, if managed well, may lead to parent communication of expectations in a supportive manner, otherwise conceptualized as a warm parental response (Wolfradt, 2002). Barge and Loges (2003) highlight two key notions to enacting a parental involvement approach to their child's development that is conducive to their success: information transmission and partnership. The researchers' findings illuminated the necessity for parents to communicate messages to their children that induce feelings of success, partnership, information sharing, and good work. Parents should foster an at-home learning environment in which students may feel welcomed, supported, and capable of asking further questions or seeking help. Here, children may feel welcome to discussing their ideas and 
opinions, asking questions, and making their own informed choices (Barge \& Loges, 2003). Holt et al. (2018) found that consistently secure, positive parental attachment can be the best indicator of a students' academic, social, and emotional functioning.

The authoritative parenting style, if managed well by the parents, may result in their enactment of a supportive style to the communication of goals rather than expectations (Ingoglia et al, 2016; Jones \& Bodie, 2014). For this research, the supportive style (positively managed authoritative parenting style) may be seen as parental communication of goals, instead of expectations, to be whatever contributes to the child's psychological well-being and fulfillment in life. Here, the parent has no direct desire for dependency nor control of the child's future decisions but rather, seeks to induce autonomy and self-fulfillment within the child's mind (Jones \& Bodie, 2014). The emerging adult may feel independence while also knowing they have parental support. Goals for positive outcomes and reflective decision making are enforced in contrast to expectations about certain outcomes (Holt et al., 2018). The prescriptive style serves as direct opposition to the supportive communication style.

Prescriptive Styles. An over-attribution of the authoritative parenting style may lead parent communication of expectations to be highly prescriptive (otherwise conceptualized as "parental demand (control)" (Wolfradt, 2002, p. 522). To conceptualize prescriptive communication of expectations, a study by Smetana (1995) exemplifies how children may develop perceptions of their parents as authoritative and limiting. Further, Wolfradt et al. (2003) explain the role of parenting styles and anxiety in adolescents. Their findings suggest that permissive and authoritative styles of parenting result in higher dysfunctional personality traits for adolescents raised in such parenting styles. Ingoglia et al. (2016) investigated two differing expressions of perceived parental psychological control. These two expressions involved 
dependency-oriented parental control and achievement-oriented parental control. The study sought to explain the pathways linking parental controls to emerging adults' internalizing difficulties. Results indicated that the more prescriptive, dependent, and achievement-oriented parental control becomes, the more internalizing difficulties emerging adults may face causing identity turbulence and self-esteem issues.

The authoritative parenting style, if poorly managed by the parents, may result in their enactment of a prescriptive style that emphasizes the communication of demands and expectations (Faherty et. al, 2020; Ingoglia et al, 2016). Here, emerging adults may not feel the authority to discuss questions/opinions, ask questions, or even feel allowed the independence of making their own decisions (Faherty et al., 2020). Instead, parents try to largely influence emerging adults' journey towards adulthood and emerging adults may feel pressured or obligated, through parental messages about expectations, overinvolvement, overparenting, and parental control, into certain careers, worldviews, identities, and/or relationships (Faherty et al., 2020). Where the supportive style of communication seeks to, verbally and nonverbally, improve the psychological state of another person, the prescriptive style occurs when parents enact verbal and nonverbal communication to direct and/or control the decisions and autonomy of their children; most often equated to the concept of parental control (Faherty et al., 2020; Jones \& Bodie, 2016, p. 371).

During the process of transition towards independence within the emerging adult's college years, parents remain a strong motivational, academic, and health influence in the college students' journey (Agliata \& Renk, 2007). In such a case, parenting styles that are prescriptive, demanding, and/or overinvolved may present detrimental outcomes on the emerging adult. Wintre and Yaffe (2000) researched the first-year students' adjustment to university life as a 
function of the relationship between parent and child. Researchers found an indirect, positive relationship between managed authoritative parenting style and the student's ability to adapt (variables). While studies, such as Aziz et al. (2011) promote parental involvement within their child's education initially, to aid in academic motivation and achievement, others, such as Kriegbaum et al. (2016) emphasize the substantial influence and investment parents have on their children and their education. Accordingly, the latter research suggests that while parents and children may share academic goals and mutually/jointly enact behaviors to obtain those goals, the parental role, if unmanaged and manifesting, may lead to a directing and controlling approach that may soon become detrimental to the emerging adult (Faherty et al., 2020; Kriegbaum et al., 2016).

As the student strives towards academic achievement and success, their perceptions of parental messages can have summative impacts on a variety of variables towards their descriptions of their own academic, collegiate experiences (e.g., academic achievement and motivation, mental health, motivation, affective distress) (Tang et al., 2018). Therefore, the potential for parental overinvolvement and/or overparenting elicited during this transitional stage become imperative to examine in order to fully understand the emerging adults' experiences in dealing with prescriptive and demanding parental expectations.

\section{Parental Overinvolvement/Overparenting}

Throughout the existing research regarding parental involvement is the assumption that parents, students, and teachers share similar notions of what may count as parental involvement with the educational context (Barge \& Loges, 2003); however, such an assumption may soon become problematic as research begins to uncover the differences in parental involvement and communication forms from parent-emerging adult during their college years. While research by 
Barge and Loges (2003) exemplifies the strong connections between positive parental involvement and students' academic success, the critical differences between types of parental involvement and communication forms (parental expectations) may have a vastly different outcome on students' perceptions of their own collegiate experience (e.g., perceptions of their own academic success and achievement).

While some children are often more focused on their education when parents are involved (Perriel, 2015), others may experience stress and according adjustment issues (Agliata \& Renk, 2007). For example, Phillipson and Phillipson (2007) state that parents' educational values and expectations can shape or change the ways in which parents interact with their children. Silva et al. (2008) found that college students performed better in college when perceptions of parenting they received throughout childhood (e.g., anxiety, motivation, and academic success) are positive. Dorrance Hall \& Scharp (2018) detail the challenges associated with the emerging adults' transition to college and the impact of positive familial conversation on the emerging adults' transition within higher education. Further research indicates that students from low conformity orientation families had positive familial conversations in college, reported higher self-efficacy, and were less lonely as they adjusted to college than those from high conformity orientation families (Dorrance Hall et al., 2017). Further, Castro et al. (2015) found that particularly high involvement and expectations (maintained and communicated from parents to students/children) were most linked to high achievement orientation and stress in students. Akhtar \& Aziz (2011) additionally found a positive correlation between parental pressure and student academic achievement. Parental pressure, via communicated expectations, placed a higher responsibility on the student to perform well within their educational context. However, following such high expectations, college students may still seek parental approval 
within their academic efforts to try to meet perceived expectations and thus experience heightened stress and/or adjustment difficulties (Agliata \& Renk, 2007; 2009). Thus, despite positively correlated academic outcomes for the collegiate student when parents are positively involved, overinvolvement and the intensity and pressure of expectations communicated may also serve as a major source of affective distress (Agliata \& Renk, 2007; Faherty et al., 2020).

Perhaps one major source of overinvolvement and overparenting for college students may be parental manifestations to help direct and guide every aspect of the collegiate student's decision making and college experience. Though falsely perceived as a heightened level of parental affection, helicopter parenting may soon lead overinvolvement and overparenting within the parent-college student relationship and, in turn, out of expectations for reciprocity, lead to manipulation (Hesse et al., 2018). Helicopter parenting is defined as excessively involved parenting and inclusive of inappropriate tactics meant to withhold the autonomy of the child through control (Padilla-Walker \& Nelson, 2012; Segrin et al., 2012). As a result of controlling behaviors, collegiate students are stripped of their ability to establish autonomy and may experience many difficulties within their college experiences due to a dependence on parental input. For example, Miller-Ott (2016) researched the effects of helicopter parenting on a college students' willingness to engage in out-of-class communication with faculty. They found that students experiencing controlling behaviors from their parents may result to turning to their parents for academic guidance instead of their teachers, thus putting their academic performance at risk.

The reviewed literature suggests that as a result of overinvolvement, overparenting, helicopter parenting, the college student may face a variety of adverse outcomes. Parental involvement/overinvolvement may entail many different facets (e.g., helicopter parenting) and 
may impart many different difficulties within the college student's experiences (e.g., affective and motivational distress, lack of autonomy). At the collegiate level, overinvolvement and overparenting within the parent-college student relationship exists where parental desires to be enmeshed with their children, through an overemphasis of expectations (Agliata \& Renk, 2017) and connection (Lynne et al., 2017), may soon lead to problems regarding parental demands and control (Hesse et al., 2018; Lynne et al., 2017).

\section{Parental Control}

When the tipping point of the parental paradigm between supporting and respecting an emerging adult's autonomy falls more towards parental manifestations to support via communicated prescriptive and demanding pressures to "obey, think, and feel in specific and subscribed ways," psychological control may become of concern within the parent-emerging adult relationship (Faherty et al., 2020, p. 697). Here, parents fail to practice messages that allow for autonomy and independence, and instead, practice messages that indirectly enforce expectations and inhibit the personal development of the emerging adult (e.g., privacy invasions, obligation, guilt, shame, silent treatment).

Tao and Hong (2014) suggest a meaning system construed through messages about expectations from parents in which children feel more obliged and responsible to succeed academically not for themselves, but for their parents. According to Agliata and Renk (2007), students often enact goals based on perceptions of parents' communicated expectations for their academic success. Here, academic achievement as an obligation is the difference between "having to" and "wanting to" succeed and achieve (Wang \& Heppner, 2002). Another tool used to achieve parental control via message communication may be guilting. As Johnson (2018) presents, "guilting is shown to be used as a tool to control others' future actions" and thus may 
be seen as a form of the prescriptive style to communicating expectations from parent to child (p. 209).

When expectations and actions no longer align with the previously co-constructed reality between parent and college student/emerging adult, guilting in the forms of complaints and assessments may occur (Johnson, 2018), with the purpose of making the person feel regret and then trying to make amends and/or change future behaviors. When guilting is used as a means of inflicting regret or self-doubt for decisions against parent expectations, the emerging adult may seek to rid themselves of the tension and discomfort by either giving in or altering decisions to align with parental expectations (Johnson, 2018). Here, guilting is enacted as a form of communicated parental control that seeks to guilt the emerging adult into continuously conforming to parental expectations for actions and behaviors.

In addition to guilt messages, parents may also enact messages of shame, as they help socialize young children and maintain control over adults (Planalp et al., 2000). Shame and guilt work together to alert the individual that social bonds have been disrupted, and thus, move the individual to take measures to repair. According to Planalp et al. (2000), guilt messages are most often enacted to induce action changes, wherein shame messages are enacted to induce feelings of self-failure. Here, the emerging adult is to feel shame for having failed to meet certain behaviors and thus may be perceived as personal failure. Guilt works to enforce social obligations while shame works towards social expectations (Planalp et al., 2020). Within the parent-emerging adult relationship, guilt messages work to induce actional adherence to parental expectations, where shame messages work to induce feelings of failure when adherence and fulfillment (of parental expectations) is not attained. 
When parents' expectations are not met, they may use the silent treatment to communicate disappointment (Rittenour et al., 2019). Silent treatment is the enactment of restraining and withholding verbal directive (messages) from the other person as a dark sided behavior seeking to manipulate or change behavior/actions without stating the desire outright. Rittenour et al. (2019) describe how the silent treatment, enacted via aloofness, disengagement, and avoidance can lead to frustration and discomfort in the receiver that ignites a desire to alter behaviors and actions to align with the desires of the user. Within the parent-emerging adult relationship, the silent treatment is used as a covert and overt means of communicating disappointment/disapproval to encourage the other to engage in change behavior. Here, the parent may enact silent treatment to cause the emerging adult discomfort or frustration within their decisions to pursue personal autonomy and independence until, finally, succumbing to parental expectations and changed behaviors. In opposition to the silent treatment, parents may also enact messages of verbal aggression and argument (Infante et al., 1994). In this context, verbal aggression and argument served as a means to communicating disagreement on terms. In either case, silent treatment or verbal aggression/argument, the ultimate goal is persuasion of the other towards changed behavior and action.

At the core of parenting styles, overinvolvement/overparenting, and parental control are the types of familial communicative patterns practiced and the resounding impact of messages elicited from parent-college student during the emerging adult's transitional stage. In the following sections, I detail Knapp et al.’s (1981) memorable messages framework that guides this study. 


\section{Memorable Messages}

Memorable messages are verbal messages that people remember for a long time and that have a major influence on their lives (Knapp et al., 1981). The basic nature of memorable messages magnifies an important implication on the parent-child relationship. Knapp et al. emphasize when messages are remembered, the reasons are complex and have to do with the recipient, sender, and message entities itself. Memorable messages are most often received from people of higher status and who are older than the recipient. Berne (1964) (as cited in Knapp et al., 1981), argues that these messages become a sort of "script" of people's lives. More often, this "script" is formulated heavily by the messages and injunctions elicited by parents such as "College is for eggheads," or "Marriage is a trap only fools fall for" (p. 38). While some messages may be ignored or belligerently disobeyed, others may have a longer impact and work to guide or explain the trajectory of the recipient's life. Thus, the accumulating evidence on memorable messages suggests that an individual forms the foundation of their self-concept from the construal of the recalled message (Knapp et al., 1981).

More often, messages include advice about how the recipient may better their life, get along with others, or pursue a certain education or job, and people can recall messages they received as early as age five. Therefore, the implications on the parent-child relationship of memorable messages becomes quite pertinent to this thesis. According to Knapp et al. (1981), the content of memorable message was found to entail mostly ideas of what one should or should not be doing in certain life stages. During the emerging adult's transition to college, feelings of unsureness and crisis amidst change may remind students of certain memorable messages. Here, the messages parents communicate may either help or hurt the emerging adult's ability to cope and or adjust. Therefore, messages that communicate actional advice or that demand attention 
from parent to child may be notable, influence the child's life, and be remembered for a long time. According to Ledbetter and Beck (2014), as children emerge into adulthood, the perspectives, influence, and beliefs continue to influence their lives and social development. Wang (2014) states, due to the variety of messages that parents communicate to their children into adulthood, parents may not be aware of their message's impact. Wang found that children may "ignore and forget some of the messages they hear, but they may also remember, accept, and internalize [some messages]" (p. 270). Wang concluded that parental messages play a significant role in emerging adult's perceptions about their self-concept and the ways in which they manage themselves in college.

An overarching conclusion in the cited research is that parents' communicated expectations may be detrimental to emerging adults in college. The types of messages parents communicate to their children throughout development and into adulthood have high implications on not only the adult's self-concept, but also their ability to make independent and autonomous decisions. As exemplified in a study by Stipek (1998), when parent messages demand attention or call children to act in certain ways, the emerging adult is likely to feel parental control and/or the obligation to fulfill parental commands/desires via parental messages that elicit pride, shame, or guilt. Thus, the types of messages and the construed meaning systems of parental messages to the emerging adult surrounding the collegiate experience are highly relevant. More importantly, the persistence and memorability of certain types of parental messages may have higher implications for the emerging adult's perceptions of their own collegiate experiences and of their sense of self. Due to the fluctuating nature of the college students' transition to college between parental support and autonomy, parental messages that provide direction and advice become more memorable (Stipek, 1998). Here, the responsibility on 
the parent to communicate supportive messages over prescriptive messages becomes imperative to the emerging adult's ability to cope and manage life turbulence and change throughout their transition to adulthood.

At the core of parental messages of expectations and control are the resulting internalizing difficulties the emerging adult may face towards their perceptions of their college experiences. Wang and Hepner (2002) argue that parental expectations, and the stress of having to live up to them, may be a key indicator of emerging adult psychological stress. Ingoglia et al. (2016) studied the internalizing difficulties emerging adults face as a result of parental control, permissive parenting, and psychological stress. During a time when parents are supposed to step back and allow the emerging adult their autonomy and self-decisions, instead, they induce pressure to conform to expectations via love withdrawal, shaming, and guilt induction (Ingoglia et al., 2016). Permissive parental/psychological control may then take on two forms: dependency-oriented control and achievement-oriented control. Permissive parental control thus inhibits emerging adult development of autonomy and impairs their ability to engage in identity developing exploration. Feeling a lack of control may lead to furthered feelings of anxiety and criticism of the self as a "failure" towards meeting parental standards (Ingoglia et al., 2016).

If parental expectations influence college student experiences and academic achievement, research investigating the various impacts messages about high/unrealistic parental expectations becomes necessary to illuminate the differing, potentially detrimental, outcomes on the collegiate student who internalize these messages about expectations. In the following sections, I review a few of the various researched outcomes of parental expectations on collegiate student motivation, mental health, depression, burnout, and persistence. 


\section{Parental Expectations and College Student Outcomes}

Perhaps the best path towards understanding why discrepancies between parents' expectations and college students' perceptions of their parents' expectations may cause such adverse outcomes and emotional turmoil is via Higgin's (1987) self-discrepancy theory. The cognitive theory was developed to explain the phenomenon wherein conflict among the ideal self (attributes about the self the individual wishes to possess), the actual self (attributes that individuals possess currently), and the ought self (attributes that the individuals believe they should or are obligated to possess) results in negative emotions (Agliata \& Renk, 2007). For the emerging adults in college, conflict between the actual self and parental expectations for the ought self, as well as the ideal self and parental expectations for the ought self, may cause the college student to experience certain perceptions of their overall college experiences and their own academic success and achievement. In the following sections, I detail researched examples of certain perceptions college students experienced as a result of parental expectations: motivation, mental health, depression, burnout, and persistence. These examples illuminate a few of the various themes that have emerged from the research on parental expectations.

\section{Motivation}

Academic motivation for college-level students relies highly on the parenting style used (Tang et al., 2018). In a cross-cultural study, Tang et al. (2018) found that while authoritative and authoritarian parenting may be positively related to autonomous motivation in Chinese families, in Western families, such parenting may be perceived as controlling and restrictive and is usually characterized as undermining to children's degree of autonomous motivation. Within their chapter on self-determination, Grolnick et al. (1997) describe how, in an effort to possess societal and parental value, children will voluntarily act in accordance. Aligning with self- 
determination theory, researchers distinguish two types of motivation: intrinsic and extrinsic. Whereas intrinsic motivation includes spontaneous and underlying behaviors innate to the individual, extrinsic motivations are derived from external reward outcomes, external demands/expectations, maintenance acts, or the belief that the outcomes may be important for mental or physical health. Rostami et al. (2015) studied external factors that predicted a student's motivation to study a second language. Within their work, researchers found that students' motivational character to be the result of a variety of variables including family influence. Within the family influence context, positive family involvement had a positive impact on student motivation. Olusiji (2016) found that students were more intrinsically and extrinsically motivated towards learning when parental, peer, and teacher encouragement remained positive and supportive. However, Rostami et al. (2015) also indicate the relevance for research that seeks to understand the impacts negative familial involvement can have. From the perspective of differing racial backgrounds, Desimone (2001) details the importance of a positive and supportive family background to facilitate a student's academic motivation and success.

However, Sansone and Smith (2000) suggest that while intrinsic and extrinsic motivation work together within a self-regulatory system, the interest enhancing measures of these motivations must also be understood. While intrinsic and extrinsic motivation operate together, the "fueling" system comes between a difference of "having to and wanting to" (p. 343).

Often times, parental involvement and communication styles may serve as a negative source of both types of motivation. For example, Tao and Hong (2014) exemplify the high prevalence of academic motivation construed as an obligation within the Asian culture. Here, while intrinsic motivation may be the individual's own desire to advance in society, extrinsic motivation is construed as an obligation to meet parental expectations (external demand). The 
difference for these individuals lies between the idea of "having to and wanting to" (Sansone \& Smith, 2000, p. 343). In their study between Western and Asian cultures, Basar (2018) further highlights the differences between academic motivation within Western versus Asian cultures. In the former, academic motivation is often a function of choice and action for the self, where "they achieve better outcomes, presumably because these satisfy students' psychological needs for autonomy, competence, and relatedness" (p. 7). However, when academic motivation is a function of a "sense of duty or obligation" and action for the other, "there may be some problem in applying the concept of autonomy to these populations' (ex. Asians) (p. 8). The overarching findings of this study and the literature reviewed indicate the existing reality that supportive parental messages and familial involvement may serve as a positive source towards both intrinsic and extrinsic motivation. However, prescriptive parental expectations and communicated demands (however unrealistic) alongside negative familial involvement may serve as a source of extrinsic motivation wherein students/emerging adults internalize such memorable messages and begin to act for the other instead of the self; paving the way for furthered negative outcomes.

\section{Mental Health, Depression, and Burnout}

Collegiate students who experience positive, supportive, and caring involvement with their parents suffer less anxiety, depression, and academic stress than those whose parents are overly involved, prescriptive, and permissive (Barton \& Hirsch, 2016). In addition, collegiate students who received new or continued informational and supportive involvement (from parent, peer, or teacher) indicated that the positive association decreased academic stress and depression (MacGeorge et al., 2005). Thus, collegiate students who are allowed autonomy and self-oriented goal setting, within well-managed parental messages of support (authoritative parenting style; warm with some control) are better able to adapt within the collegiate environment and manage 
their own path to adulthood without experiencing mental or physical concerns. Wang and Hepner (2002), in their study of Taiwanese students, found that meeting parental expectations and having the duty to fulfill them was positively correlated to affective feelings of psychological stress. When actual actions did not align with parental expectations, students reported feelings of failure and shame. Miller and Day (2002) found that female college student suicidality was positively associated with parental expectations of family conformity and perfection. In a study on collegiate student burnout and the antecedent conditions that may impose such a condition, Cushman and West (2006) found that (among others) outside influence and suffering mental/physical health influenced collegiate students to "drop" or "burn" out. Parental/familial demands and unattainable expectations were among some of the "outside" influences defined. Therefore, discrepancies between college students' behaviors and parents' expectations may predict college students' anger, depression, and anxiety (Agliata \& Renk, 2009). While the research reviewed focuses more on the negative outcomes of parental expectation and unrealistic demands, despite many negative outcomes, the outcome of persistence may be dark sided in nature yet serve as a fundamentally positive outcome for the emerging adult, collegiate student.

\section{Persistence}

Both parents and teachers influence the goals that the emerging adult may set for themselves in college (Ritzer \& Sleigh, 2019). While negative parental involvement may lead to negative health or psychological problems, positive parental and teacher involvement has been documented to lead to persistence towards goals and priority setting. Additionally, according to Wang and Nuru (2017) when parental goals and personal goals align, student persistence is further facilitated. Research has yet to examine what factors cause the emerging adult (who received and internalized prescriptive and controlling messages) to still pursue their own 
autonomy/independence and persist in this manner. However questionable the prescriptive, demanding, overinvolved, and controlling parenting styles may seem, the context itself may serve as a positive source of perseverance or persistence in the emerging adult, thus representing the light and dark-sided natures of parental messages about college.

\section{Research Questions}

During the emerging adult's transition to college, moments of vulnerability and adversity serve as opportunities for identity construction and autonomy/independence. At this level of development, parental provision of developmentally appropriate support and warmth allows the emerging adult the opportunity to adapt within their adult role in a more successful manner. However, if parental involvement, messages, and expectations become manifested within prescription, control, and demand, the collegiate student's perception of their college experiences may present a variety of positive and negative outcomes. A memorable messages framework was used to illuminate how and why emerging adults recall certain messages and adhere (or do not adhere) to parental authority and decision making during this critical stage. The resonance of parental messages provides a context within which unmanaged parental statements of high or unrealistic expectations may be detrimental to the emerging adult's perception of their college experiences and their own academic success and achievement. Here, outcomes regarding motivation, mental and physical health, depression, burnout, or persistence may impact the emerging adult's ability to perceive themselves as academically successful and achieving. Thus, in this thesis, I examined significant and long-lasting recalled parental messages about expectations that emerging adults in college receive(d) from their parents. I posed the following research questions: 
$\mathrm{RQ}_{1}$ : What memorable messages do college students recall receiving about expectations regarding college?

$\mathrm{RQ}_{2}$ : How do emerging adult college students respond to parental messages about expectations in college?

$\mathrm{RQ}_{3}$ : What are emerging adult college students' perceived outcomes of parental messages about expectations in college? 


\section{CHAPTER III: METHODS}

Qualitative research is fundamentally motivated by curiosity expressed in questions that seek to understand human symbolic performances and practices in communication (Lindlof \& Taylor, 2019). Qualitative research design was the best approach for this study as the research questions sought to develop robust accounts of participants' stories. With the complexity surrounding parent-child relationships, in-depth, qualitative interviews were the most appropriate for this study to uncover the nature of a person's experience and perceptions of their own realities.

\section{Participants}

In-depth, qualitative interviews were conducted with 14 people who identified as having received, or who still receive, messages from their parent(s) or caregiver(s) about college, success, achievement, majors, jobs, and/or priorities. Criterion sampling was used to ensure participants met the terms of the research study (Lindlof \& Taylor, 2019). To qualify for this study, participants had to be at least 18 years of age, remember receiving messages from their parent(s) or caregiver(s) about college, and believe that these messages have influenced their college experiences in some way while also being comfortable speaking about their experiences. Before the recruitment of any participants for this study, IRB approval was obtained. Participants' ages ranged from 20 to $35(M=24)$. Nine participants were female and five were male. Participants self-identified their race, and nine were Caucasian, one was Hispanic, three were Black/African American, and one was Asian (Indian). Thirteen participants were born in the United States, and one was born in Germany. Ten participants' parents/caregivers birth country was the United States, two were the United States/Germany, one was Greece/Canada, and one was India. Eleven participants identified as graduate students, two were juniors in 
undergraduate college, and one was pursuing their Associate's degree. Thirteen participants reported on their biological parents, and one reported on their biological mother and grandparents (mother's side). All fourteen participants reported living with their caretakers for eighteen years (their whole lives) before "going to college." Participants reported recalling messages that began as early as the age of 3 and as late as $19(M=12)$. Eight participants communicated with their parents every day, five every week, and one communicated with their parents once a month. Of these participants, four participants shared that both their parents had college level degrees, six shared that one parent had a college level degree and the other did not, one shared that their mother had a college level degree and their grandparents did not, and three shared that neither parent had a college level degree.

\section{Procedures}

Participants were recruited through the Illinois State University communication research board (see Appendix A for research board recruitment). Additional recruitment/study information was obtained through posts to the researcher's email and Facebook page (see Appendix B and Appendix C for email and Facebook recruitment). Recruitment materials sought participants who specifically recalled parental or caregiver messages about college and who believed those messages have influenced their college experiences. To participate in the study, participants were required to contact the researcher directly through email. Upon contact, the researcher responded with the requirements for participation and a consent form to be read before participation in a scheduled interview. Participants were made aware that they may exit the interview at any time and choose not to answer any questions. Such protocol was explained in the initial email as well as within the consent form. Before beginning the interview, the researcher read the consent form aloud for each participant (see Appendix D for informed 
consent). To allow participants to guide the interview, I utilized an interview guide as opposed to structured questions (Lindlof \& Taylor, 2019). The interview began with questions about demographics and their parent-child relationships (e.g., What is your age? What parent(s) will you be reporting on? (e.g., biological, foster, grandparents) How long have you lived with your parent(s)? How often do you visit/speak with your parent(s) now?). After these demographic/relational questions, participants were asked about their conversational relationship with their parent(s) or caregiver (s) (e.g., How would you describe the types of conversations you and your parent(s) have? How involved would you say your parent(s) or caregiver(s) are in your college experiences?). The next set of questions focused on the types of messages participants recall (e.g., How have conversations about college taken place? What messages can you remember getting from your parent(s) or caregiver(s) in the past or more recently about college, majors or jobs, success, and priorities?). The next set of interview questions focused on participants' perceptions of the outcomes of these messages (e.g., Looking back now, do (or did) you ever consider these messages to be impactful on your overall mental health?). Finally, the researcher asked participants if there was anything else they would like to share concerning messages they recall receiving from their parent(s) or caregiver(s) about college (see Appendix E for interview protocol).

\section{Data Analysis}

The semi-structured interviews lasted between 20 and 60 minutes $(M=45)$. Interviews were recorded and later transcribed verbatim, resulting in 178 single-spaced pages of text. Pseudonyms were assigned to each participant to maintain confidentiality. Utilizing thematic analysis to analyze college data from the interviews provided a rich and detailed, yet complex, insight into participant realities (Nowell et al., 2017). Thematic analysis allowed for an 
examination of the various perspectives that participants may have on shared experiences, highlighting of similarities and differences, and the potential to generate unanticipated insights (Nowell et al., 2017). By using thematic analysis, the researcher was able to search for emergent themes across the entire data corpus allowing for the analysis of messages about college within the parent-child relationship. Braun and Clarke (2006) suggest a six-step thematic analysis. Following their iterative and reflective process, thematic analysis began with the researcher familiarizing themself with the data. Here, I transcribed the interviews and read the data through three times. Next, I generated initial codes, and then, as per the third step, I grouped the initial codes into potential themes. Fourthly, I reviewed potential themes in relation to initial codes and data as a whole before, fifth, defining and naming emergent themes along an emergent storyline. The final step of the thematic analysis occurred when I wrote a summarizing report supplemented with thick descriptions and exemplars. Throughout the analysis, concepts and ideas from memorable messages framework (i.e., how and why how certain parental messages may become more memorable and thus, more impactful on the emerging adult, college student how they respond and how these parental messages influence collegiate students' perceptions of their own collegiate experiences) were used to help make sense of the parental or caregiver messages, and to make sense of the data, establish codes and themes, and draw in-depth exemplars from interview data per participant. 


\section{CHAPTER IV: RESULTS}

Using the memorable messages framework, I conducted and analyzed 14 interviews with individuals who identified as having received (or still receive) messages from their parents about expectations about college. The transcript data were coded for emergent themes until the points of redundancy and saturation. Themes were then categorized along responses to research questions posed. Once categorized, emergent themes/storylines were narrowed for each research question posed. In the following sections, I provide emergent responses as categorized by order of research questions posed.

\section{College Expectations Message Types}

In the first research question, I sought to understand the memorable messages emergent adults recall having received, or still receive, from their parents about expectations regarding college. Participants recalled receiving three types of messages from their parents about expectations regarding college. First, participants recalled parental messages that were stated outright, as participants perceived as part of a largely agenda-based, directive conversation seeking to convey explicit expectations. Second, participants recalled parental messages that were more indirect and provided support to the child. Here, messages about college were not stated as outright expectations, but more as a part of larger, continuous conversations that sought to merely invoke thoughts about college and the emerging adult's future. Third, participants recalled messages that were direct yet still provided support and aimed to create the child's autonomy and self-efficacy.

\section{Direct and Prescriptive Messages about College}

Parents' messages that included explicit expectations often emphasized college as the only pathway towards a successful life. Children often did not feel supported or autonomous 
when receiving these messages; instead, participants recalled messages in which parents talked about college as something that they expected children to do and never question. For example, Brittany, a 24-year-old Caucasian female reported messages from her parents about college as something that was explicitly stated and directly reflective of her parents' expectations:

Yes, it was very much the expectation that I would end up in college. My, it was, I, there was talk for a little while that I was going to get a cosmetology certification, and then go to college. But like college is always somewhere expected to be in the plan.

Similarly, Isabel, a 23-year-old Caucasian female emphasized that beyond the explicitly stated parental expectations to attend college were the explicitly stated, memorable messages about success being highly predicated on the achievement of such expectations:

Early on in high school, they started talking to me about how I needed to go to college to be considered successful, and that was kind of an expectation in our family ... so like I said, in my family, college is kind of seen as a requirement for success. So, they were very over and outright with that whole viewpoint in terms of messages that I received. So even from high school, I remember receiving messages that quite literally said, you need to go to school to be successful, you need a degree to make good money. So, it was very outright in the focus that you had to go to college. So, in my family, if you don't have some form of higher education, they don't really consider you to be a successful person. So it's not necessarily how happy you are with your life and how it's going. But it's, are you financially stable? Do you have some sort of educational degree? Are you doing well in life?

Participants who recalled these types of direct messages about college reported perceiving that college was not presented as an option, one to discuss with their parents, but 
instead a decision they would make because their parents told them to do so. When recalling these types of prescriptive messages, participants discussed that they felt uncomfortable seeking out their parents' help and guidance, rendering them incapable of autonomy and self-efficacy. The way that many participants talked about their parents who used these types of messages seems to reflect an authoritarian parenting style, characterized by high demand and low responsiveness. For instance, Elliot, a 35-year-old Caucasian male emphasized his collective struggle with explicit parental messages communicating expectations and demand:

During my undergrad, I was living with them. And it was just a really different time, we grew up in a very, very, very, very strict, evangelical household. And even in college, there was this expectation that the parents made all the decisions. All of them. And it wasn't explicit. Because explicitly, they would say, like, oh, we want you to make good choices. And but if the decision, if your choice was not good, then it was sort of made for you. Right. And so, yeah, my decision making was wildly controlled by that, I would say, back then. But there were externalized expectations that you go to school, and then you come home, and you don't really do anything in between . . . and like control. But I would say that they're [messages] super consistent with how I was raised. You know, the way I was raised was his sort of overbearing, controlling. They're issuing edicts and proclamations about your life, and you're expected to follow those. And I think that's really consistent with how their communication about education was.

Emphasized by Elliot's talk are the implications of explicit, parental messages about expectations in college that maintain control over children while limiting autonomy. Gloria, a 23-year-old, female identifying Black/African American also emphasized the negative influence of her recalled parental messages: 
So just throughout the college career, I just recall specific things of her being like, keep pushing, you know, keep going, you got this and I love that she's like that, but sometimes I would I was just wishing that her messages would be more like my dad's where he was like, just breathe, take a break or you know, like, just go breathe. Or, you know, he would say things like, you know, I know college isn't for everyone. But you're doing great. So you know, like, even if you take a break from school, just make sure you get what you want. You know, and my mom was just never really she never laid out options for me. It was just school is the option for me. So just those kind of conversations. Or like, if I, if I ever mentioned to her like I don't want to I don't want to get anymore, I already know that that conversation would just be like, oh, she probably lose her mind and just keep keep trying to talk me into it instead of trying to understand why this isn't working. She would just constantly tries to talk me back into it, like, keep pushing. You got this, or just, just this constant reminder of like, I guess like she doesn't know what else to say besides keep going. And I guess that's not enough for me. Why keep going? You can't tell me why I should keep going. You're just saying keep going.

Gloria's family experiences and recalled messages further emphasize the mental and emotional strain parental messages about direct expectations may impart. Gloria and Elliot's talk illuminate the collective struggle faced by participants who perceived their parents' messages to be heavyhanded, direct, and often controlling.

Overall, these recalled, explicit parental messages about expectations seemed to affect negatively the children's self-concept and efficacy. For instance, in Elliot's situation, in lieu of his parents' rigid expectations, Elliot was rarely allowed to make choices about his own life in many contexts, including education. For Gloria, burdensome parental messages imparted heavy 
expectations and emotional strain while additionally limiting her ability to make her own life choices. In the next section, I discuss recalled messages, conveyed implicitly, through which parents were perceived along the supportive parenting style.

\section{Indirect and Autonomy-Supporting Messages about College}

Data from my interviews suggest that parents did not always convey expectations in a heavy-handed, directive manner. Many used more indirect, albeit strategic ways (as perceived by participants), to communicate their expectations. Some participants described their parents as highly supportive and autonomy providing when it came to making choices about college. Participants recalled messages about college that were often part of larger conversations about life choices that emerging adults framed as open dialogue. While recalling these types of implicit messages, participants explained how they not only felt comfortable seeking out their parents' help and guidance, but also felt that they have self-efficacy and autonomy to make their own choices. For example, Aaron, a 24-year-old Caucasian male emphasized how his parents never explicitly stated that he must attend college; rather, he believed that they collectively shared their college experiences as a way of motivating him to attend college. Here, while his parents might have communicated their desire for him to attend college, they never explicitly stated their expectations:

Not so much like talking about me to go to college, but more so like, we talked about their college experience. Like, that's always been a part of my family history so that's always been a part of college I guess? Um so, my parents both attended the same school. And growing up, we were big fans, like we'd watch the sports events of the school for this university and kind of like a community identity around it. And I was always excited to go to this specific school for a while more so than even just the idea of college is the 
idea of joining my family tradition of going to this specific University. And then it wasn't even a question or something we talked about necessarily, like, am I gonna go to trade school? Or am I gonna go to college? It was already, it was almost like, I definitely think it was the expectation. So that kind of growing up with tone and expectation of being part of the family of going to university and then also the assumption I think that if you wanted to provide for a family you needed to go to college, if you want to be successful, if you want to have a job. That was kinda a family identity thing honestly, like we are the [family last name], we go to college. This is like, this is the kind of life. The assumption in the family was doing the college is the way of having success.

While the messages Aaron received from his parents about college never directly stated his parents' expectations, the underlying perception was college as an implicit expectation. In this fashion, messages were never construed directly, nor heavy-handedly, but rather, as part of a larger conversation about family history, and inevitably, college as a pathway to success and identity.

Quite a few participants talked about their parental messages as implicit, yet they believed the messages were strategic to motivate them to do what the parents wanted. Chloe, a 23-year-old Caucasian female emphasized how although her parents were not direct with their expectations through their messages, the way that they talked about college was memorable:

I remember my parents, they never said you have to go to college. Like that was never something that I remember them telling us. I remember them saying, like, you're smart enough to go to college, laying out the opportunities that college provides you with. But if you wanted to go to trade school, like my dad did, or if you wanted to go to beauty school, or whatever, like they were always supportive of that. 
However, as Chloe continued her explanation, she shared that while her parents provided multiple options at first, supportive of any outcome, the underlying expectation seemed to be that she attended college, but they did not push that option on to her. They wanted her to reach that conclusion on her own. She said:

And I think once my like my older sisters went to college, it was less of a standard that was presented by my parents and more just a, almost like an expectation within our little ecosystem of a family that we went to college. I don't ever remember myself personally, like not wanting to go to college, it was always something I wanted to do. So I'm not sure if that was, those feelings were facilitated by the conversations that I've had with my parents, but I know that it was never pushed upon us as good ... I think they were probably strategic in the way they were talking to me about them. I think they probably in their mind, were like Chloe should go to college. And we want her to get good grades, and we expect her to be successful. But they were probably strategic in the way they verbalize that to me. So instead of saying that, they would say like, you know, you have really good grades in school you like going to school you enjoy learning, college is a great place to build upon certain educational pathways or majors, I guess, that you're really interested in. So I think, purposefully. verbalizing college as like, an opportunity for me and like a really great opportunity as opposed to verbalizing it as an expectation and something that I should do is probably what I think was their collective angle.

Aaron and Chloe's talk illuminates that while parents may still have expectations that children go to college, they may choose to convey those expectations through implicit messages that allow children to reach the conclusion to attend college on their own. In these cases of implicit messaging, participants described their parents as supportive and never controlling, unlike the 
participants in the previous section who seemed to describe parents more characteristic of authoritarian parenting. Children who received implicit messages believed that they were able to engage in conversations with parents about college. They felt more autonomous, capable of asking for help, and self-efficient. For example, later in the interview, as Aaron spoke of his parents' responses to his decision making and support seeking, he described them as never directive and always supportive of his own outcomes:

They're, they're super supportive. I don't think they've ever once said, like, maybe you should do something else, I don't think they've ever said that. I think they just said more power to you. I think they also see it. I think they also agree with me, that they, they know me well, this fits me well, that why would I do anything else?

For emerging adults like Aaron and Chloe, while college may have been the parental expectation, messages about college were guided by parental desires for their children to "do their best," "feel fulfilled," and have "a better life," but still go to college. Here, messages were more often part of a larger conversation seeking to invoke attending college as good, but still something the child could elect to do on their own. Comparatively, direct messages about college seemed to connect expected college attendance with success. These findings further emphasize the various impacts of differing parenting styles and communicated expectations on emerging adult outcomes in college.

\section{Direct but Autonomy-Supporting Messages about College}

Across the corpus of data, it appears that parents' messaging about college may best be communicated in ways that children perceive as both direct about parental expectations but also conversational and supportive. Some participants described their parents as having clearly stated expectations about which they were open to having dialogue with children. Participants 
described these parental messages as demonstrating a healthy balance between supportive and directive in an autonomy providing manner that seemed more attainable. Dejah, a 24-year-old Black/African American female, often spoke of her parents as strict but overarchingly supportive. She said that her parents were firm where it mattered to support her achievement of autonomy in adulthood. In doing so, Dejah accurately narrates how supportive yet direct messaging about college led to her perceptions of her parents' college expectations being attainable:

My parents insisted that I, first of all, go to college, and then to a university with dorms, because they knew that if I chose one of the colleges, like in town, and I lived at home, they wouldn't let me grow up. And they knew that they would still tell me what to do, because I lived in their house and would have to follow by the rules. So they actually insisted that I leave my house so that I could learn how to make my own decisions, and still look to them for advice, but not rely solely on them for advice. So they very much did want me to grow up.

While the direct parental messages may have seemed harsh as received, overall, Dejah perceived these messages as necessary for her firm release into autonomy and self-efficacy. To Dejah, with this firm, directive push, her parents helped her to gain autonomy in ways that were memorable to her:

There are times when I have looked for advice, and it was a low stakes situation. Low enough that they were just like, we're not going to tell you, you know, what we think that you should do. Because they're gonna be times when you have to figure it out. And I've gotten a little frustrated at that. But then later, I look back and I was like, okay, but I really did need to figure out what that is on my own. 
Dejah's example also illustrates how children who perceive their parents' messages to be supportive may also feel welcome to continue seeking support and guidance without judgement. In Dejah's case, where her parents were also directive, their support came in a manner that pushed her to engage in autonomous decision making. In another interview, Nyesha, a 20-yearold Black/African American female emphasized that, without her parents' supportive yet direct messaging about college, she would not have gained realistic experience and been open to growth in response to criticism:

I feel like I'd be more stuck and be like, and I can, I actually come to my parents and tell them about my college experience or what I'm going through. So even though sometimes when I am like you're a little too involved right now, it's kind of I'm kind of glad they are involved in my life and wanting to know what is going on and wanting to help me grow as a person.

Dejah and Nyesha's talk suggests that despite parents' talk being seemingly heavyhanded at first, children perceived their messages as constructive and positively influential on their autonomy and self-efficacy development. Participants who perceived their parents' messages to be direct yet supportive, often expressed that despite their harsh immediate nature, parental messages about college were often clearly stated expectations, open to dialogue, and overarchingly sourced from a supportive and autonomy providing position seeking to induce self-efficacy within their children.

It is important to note that some participants recalled messages about college that were situated within cultural expectations. Although these examples did not fit neatly into one of the message types above, they are important to address when considering the content and 
perceptions of messages. For example, Hillary discussed the push toward education in the Western culture:

I think there's always been an expectation that I would go to college, because we're very much in a society where you need to get a college degree to be successful. And that, that's kind of a very big expectation and narrative in Western culture. And so, I think that that's kind of a typical, I think, kind of a typical expectation for, for parents in the 21st century, right now, within Western culture is that kind of, well, you know, you got to get a college degree to have a good career. And it's a very kind of clear trajectory. So, I think that's kind of where a lot of their motivation is coming from.

Gloria provided a thoughtful explanation for the ways that her parents' beliefs about gender and race played a role in messages they gave her about college:

But for me, me not going to school was not an option, because to them, they see it as gendered. Or they say that men cannot, men cannot afford to go to school, because they can weld, they can go to chemical plants, like, but you're, you're a woman, and you have to go to school, you don't have the option of what your brother has, where he can go, go to Exxon and make what she would make with the two degrees. So that was another big pusher for them, is you're a woman, and you have to have a degree. So they kind of put that fear appeal of like, if you don't go, this is what you have ... you know, abide by what they are saying, and that I would just become this very successful young woman who's very independent. So that they, again, with minorities, so that when they get old, I can take care of them. So I know that a lot of other I don't know, I'm from what I've heard, and about the other minority groups that I've been around is that you go to school, so that you can take care of us when we get older. And that is essentially what I think 
they're pushing so hard for me is because essentially, I will be the one to take care of them and not my brother ... you're Black, you're female. And you have to go to school, you know, like, that's the only way to get from middle class to upper class, that's the only way to set yourself up. You can't go to school like these, with these white kids. You know, even as a child, I'm pretty sure other minorities have heard this talk to where it's your brown, like, you're Black, they're watching you, they're waiting for you to fail. So you have to be on your P's and Q's. Because they're gonna find anything to kick you out. They're gonna do anything they can to make sure that you don't succeed. Sorry. So like, things like that, um, you know, and then having the pressure of your parents that because you're Black, or because you're, you know, a minority, you have to meet this quota. versus when I talked to like, some of my, like, one of my white friends that I had, we fell out because she just couldn't understand.

Gloria's example, among others, illuminates the unique and even more salient context cultural variance may present for the formulation and strength of parental expectations and the resulting direct or indirect messages received.

Research question one sought to understand the memorable messages emergent adults recall having received, or still receive, from their parents about expectations about college. While memorable messages framework might suggest that messages will be directly recalled (i.e., the exact content shared during interactions) and thus, impactful, this research suggests that while some messages may be directly and specifically recalled, individuals may also recall messages that were perceived as more indirect (i.e., not specific messages word-for-word) that were communicated repeatedly as part of larger types of conversations about future plans and goals. Participants in this study recalled both direct and indirect messages from their parents about 
expectations about college. Direct, prescriptive messages often included the perspective that the children will go to college to be successful, with little to no input from their children. Children often framed their parents' overall communication as similar to how they talked about college controlling and not open to the child's input. On the other hand, participants who described their parents as supportive, overall seemed to recall more memorable messages about college that implicitly invoked conversations about college goals and emphasized expectations for the children to achieve happiness/fulfillment in whatever choices made; however, many participants who recalled these types of messages believed that parents were strategic in framing college as an option, but one they believed that children should choose. The third type of messaging about college expectations seemed to reflect direct communication that participants perceived as supportive. In these types of messages, parents were clear about their expectations for children to attend college but took a more constructive approach to helping children develop autonomy and self-efficacy. Lastly, I pointed out that some participants believed that their parents' messages were based on cultural expectations about college and success. The second research question of this study sought to further understand the types of responses emerging adults communicated when parents conveyed their messages.

\section{Emerging Adult Responses to Parental Messages}

In the second research question, I sought to understand how children responded to their parents' messages about expectations about college. Participants recalled responding three different ways that often differed based on parents' message types. First, children whose parents were direct and prescriptive tended to respond through compliance or avoidance. Second, participants whose parents were indirect and supportive tended to use autonomous responses.

Third, participants who received direct, yet supportive messages responded with autonomy and 
selective compliance. The following sections detail the varying types of participant responses to parental messages about expectations about college.

\section{Compliant and Avoidant Responses}

In some instances, children who received directive and prescriptive messages communicated compliance to their parents and tried to avoid further conversations about expectations. Many participants talked about just giving in and going along with parents' plans for them. For example, Gloria explained, "Um, I mean, I went with it . . I accepted that those were the expectations that I needed to meet." For some participants, like Elliot, compliance was expected because parents were seen as the decision makers:

Again, undergrad, I, I sort of chiefly bought into the communication idea of back then that they were they were the parents, so they got to choose. And sort of this family, patriarch and matriarch, but they get they get to do what's best for the family, which includes you, you know. And so, I would do what they said. Which were usually sort of broad topics. Like, what, what school you're going to, or what, what you'll study, that kind of thing.

At the core of compliant and avoidance responses was each emerging adult's initial trepidation to voice their own desires, out of fear of negative parental response and combative communication. Elliot described a sort of sibling agreement to offer "best version" responses to their parents to avoid particularly combative responses (e.g., blackmail/guilting) that would otherwise result from violated expectations:

And we describe it as sort of like the Kennedys where they have this very public face.

But then there's like, conflict and turmoil within the family. And so, I think it's sort of

like that there's this public face. And then there's what's real. And with my folks, or with 
my mom, we talked, I think a little bit more openly. But with my dad, it's very much. I wouldn't say that we lie to him. But we, we offer the most we offer his best version of ourselves in the conversation. Because it just avoids the conflicts, which would result in like I described before these sort of discussions that turn into lectures that then you either you agree with, or you know, they just like keep going forever until you sort of pretend like you agree or something.

Gloria also used compliant and avoidant responses to avoid conflict with her mother:

And like, I think that's like, the biggest problem is that, you know, like, throughout my college career, I was just making sure that like, I'm meeting this status quo for my mom, and giving her what she'd like, otherwise she's brutal about it.

Similarly, Mrudita, a 23-year old Asian Indian female, described that her mom, "gets mad when it's not her way. She doesn't like it. I know that she usually gets mad.” Knowing that, Mrudita either avoided talk about college or when her mom addressed it, went along with whatever her mom was saying.

Although many participants talked about complying or avoiding, some who received direct and prescriptive messages began to enact resistive responses that sought to intentionally violate parental expectations and allow them greater autonomy. For instance, Frank, a 22-yearold Caucasian male, whose parents wanted him to go to law school, decided that he wanted to continue to graduate school instead. He explained:

At the beginning, it was kind of like, you know, how do I please my parents was a big kind of thing that I was doing. So I didn't necessarily consult with them about what I was doing. But it was more of like that thing in the back of my mind. That was like, telling 
me, how am I going to make them happy and that kind of thing, as opposed to how am I going to be happy.

Frank chose to start forging a path that was different from what his parents wanted but avoided communication about it. Note that he made the distinction between making his parents happy and making himself happy.

Some participants chose their own paths silently while others were more forthcoming about their defiance of parental expectations. For instance, Elliot, while recalling messages from his parents that were perceived as "edicts and proclamations," described a turbulent release into autonomy as precipitated by his decision to resist and stop complying.

I think, over involvement. It was for that reason, because I won't lie, like, through the papers, and I was like, fuck this. And it was at that point that I got kicked out of the house for not accounting for every penny of my money, MY money. Which was a blessing in disguise, you know? .. . Like the expectation was you live at home until you're married. And as a gay man growing up in that house, didn't think I would get married back then. And so getting kicked out the house was like, oh, my god, I'm out now like that was that's the only other way I guess, you know. So turned out to be a really good thing.

To Elliot, without his resistance to parental messages about expectations and over involvement, he would not have achieved autonomy nor self-efficacy. Even Frank emphasized that if it were not for his resistance to parental messages, he would not have been able to pursue something he was personally passionate about:

During my senior year, I was kind of, you know, I'm going to do what I want to do. My parents, you know, can give their input, but I'm not necessarily going to listen to a hundred percent. And, you know, when I, when I told them that I wanted to go to grad 
school instead of going to law school, that was a conversation that lasted for about three hours, where I told them, you know, this just isn't where my interests are. And, you know, they, they definitely asked the questions, well, why? And they said, well, I think that you should just give it a shot, you know, that kind of thing. And I just told them, no, like, I'm not going to do that, like, this is my life, this is my interest, I'm not going to waste a year by just, you know, doing something that I'm not interested in. But I can tell you right now that I don't want to do it, doing three years or a year of law school isn't going to change my mind, it's probably only going to make that worse, because I'm just going to have the stress of it all piled on. So I think like, in the beginning, it was like, when I started college, it was geared more towards going to respect what my parents say, and kind of listen a little more in depth to what they're saying. And then after graduating, it became, you know, I'm independent on my own person, I can do what I want to do. Both Elliot and Frank had a conversation with parents about how they would not follow their rules. Although Elliot's example reflects a more highly conflictual experience than Frank's did, both decided to "go against the grain" to gain autonomy However, for some participants like Mrudita, this turbulent release did not come without more negative and combative feedback from their parents. She explained how sometimes she would reach the point of enacting even more resistant responses asking her mother to stop:

There's a certain point, I said to my mom, I should just tell her I'm like mom, like I get it, like stop. Like I get, like just stop. I don't need to hear it all the time. You know, like, about my career path, like what I'm choosing to do, and whatnot ... I'm like, we get it, you don't have to tell us all the time ... Although other family members of mine have really suffered as a result of these messages. I usually just let it go in one ear out the 
other, especially, like here so often now I'm just like, okay, I'm just gonna do what I wanted to do at this point, you can't stop me.

These participants' compliant/avoidant responses often were in reaction to direct messages about college. Participants reported the obligatory expectation for compliance to parental messages along with avoiding any additional talk or conflict about parents' expectations. However, some emerging adults fought back to enact autonomy and go against their parents' wishes but these actions often involved a fight or conflict with parents.

\section{Autonomous Responses}

Some participants, particularly those who described receiving more indirect and supportive parent messages about college, described their responses as autonomous and said that they were able to select their own paths. Because parents had not communicated strict expectations, children were not concerned with complying with parents' wishes. Here, participants talked a lot about trust in their relationship with their parents and how they never feared negative or combative parental feedback as a result of their autonomous responses. Instead, they seemed to feel mostly confident making their own college decisions. Michael even goes so far to explain that his parents trust his decision-making capability, making him feel confident and capable:

They're okay with it. They trust me. I've gotten in to good schools. I think they see me as a responsible person. So they, I would say they trust what I'm doing. And they check up on my health, I think, most of all, I think that sort of thing they get annoyed if I'm not following their advice. Other than that, they trust me. They don't express a lot of disappointment with my choices. And so yeah, I do feel confident making a lot of my decisions, at least that I can make them ... I would say even though they don't 
necessarily help me make decisions, they're still very involved. I feel like they're, they're pretty involved as far as knowing what's going on, but maybe not so much involved in making decisions and guiding what I'm doing . . . They're, they're super supportive. I don't think they've ever once said, like, maybe you should do something else, I don't think they've ever said that. I think they just said more power to you. I think they also see it. I think they also agree with me, that they know me, well, this fits me well, that why would you do anything else?

In his interview, Michael emphasized the autonomous response and decision-making ability he garnered. Chloe also shared her ability to make her own decisions due to the indirect and open messages she recalled receiving:

I think because our conversations about college were so informal. And because they were pretty like, vague, like I said, in terms of like, yeah, you can do whatever, you know, you should really think about what you're interested in, maybe you should talk with some like admissions counselors or whatever ... They really just wanted me to be happy. They wanted to let me explore the different avenues that I wanted to explore.

Chloe further explained that, "They want to support me ... I invite them to be involved in my experiences and my education. And they also like, want to know what's going on? Because I think that's, I think that's just what parents do."

Michael and Chloe's autonomous responses seemed to be communicated without fear of negative parental response or expectations of compliance. They felt mostly confident and capable of making their own decisions and being autonomous in college. Participants described parents as interested but never overbearingly invested in their college which makes sense considering children recalled receiving indirect and supportive messages from these parents. 


\section{Autonomous and Selectively Compliant Responses}

Finally, some participants, particularly those who described receiving more direct and supportive parent messages about college, described their responses as autonomous but still considerate of parental messages about expectations. Children who explained using these responses were able to articulate parents' expectations and believed that they could take parental messages into consideration, without the expectation of compliance, while making their own decisions. Participants communicated their ability to voice their own desires despite a bit of parental feedback. Although parents at first did not like the pushback, participants described their parents as generally accepting of their goals. Overall, these emergent adults feel responsible for their adult role in society with a firm, yet supportive backing from their parents. Dejah described her ability to autonomously, and selectively, respond to her parents' recalled directive yet supportive messages with confidence and self-efficacy:

I consider it just because I know that they, you know, lived full lives and do a lot and you know a lot of things. So, I take what they say to heart, and is very important to me. But I also remember that they are older now. And they wouldn't necessarily do everything that I would do. So there have been times when I've either asked for advice, or they gave me unsolicited advice. And even as they were speaking, I was like, well, I know I'm not gonna follow that, but I'm gonna respect them. And by listening to the rest of this, um, and then sometimes also like, oh, I actually have something good in there and they keep going. Or sometimes I'm just like, not sure not, not doing whatever they suggested. And then sometimes I'm like, oh, no, that's actually exactly what I needed to hear . . . But yeah, I value it, but I don't always follow it . . Um, and they help me feel successful. Because even if I don't agree with what my parents have said, or how they say said it, I 
do not come from a place of love. I do know it comes from them knowing a little bit more about the world than me. And so I use it as a motivation, of just wanting to make my parents proud. Um, and then even if I don't follow the advice, they give me the suggestions they give me, or I don't come away from a particular conversation, feeling uplifted. I just take the sum of every conversation and just thank my parents just want me to be as successful as I can be. And I use that as motivation. And then, but not just motivation. But also, what is the word I want, as like a guiding light in the conversations and not conversations and the decisions that I make. So when I make my choices about my schooling, what classes how long I'm going to be here. It's not what would make my parents happy. But it's what's going to put me at the best advantage in life. What's going to keep me sane, what's going to help me because I know that's all they want for me. Dejah's response behaviors best exemplify the sorts of autonomous and selectively compliant behaviors emerging adults may enact when they perceive communicated parental messages as direct yet supportive. Like Dejah, most participant responses to directive yet supportive parental messages acknowledged the parents' concerns for them but also reflected the child's ability to take their parents' information and suggestions and make their own choices. For Nyesha, while her parents never stop her from making her own choices, she always seeks their feedback:

For me, personally, I like to make my own decisions. But I have it in the back of my head that maybe I should consult with my parents first. So I am one to go talk to my parents first. Even after like small things, let me call my mom and FaceTime her and see how, what does she think about it? So I have to say I do get my parents input sometimes before I make a choice that seems kind of sometimes like a very big life decision. I just have like this gut feeling that maybe I should go tell my mom really quick. Maybe I should ask my 
dad, maybe I should do this. It's like, let me go ask my parents before I do something out of routine ... Maybe they know better? Because they're older than me, wiser than me. They've been through something.

In summary, research question two sought to understand the types of responses emerging adults elicit to communicated parental messages about expectations about college. Participants recalled responding three different ways that often differed based on parents' message types from research question one. First, children whose parents were direct and prescriptive tended to respond through compliance or avoidance. Second, participants whose parents were indirect and supportive tended to use autonomous responses. Third, participants who received direct, yet autonomy-supporting messages responded with autonomy and selective compliance.

Collectively, emerging adult responses indicated that they wanted more autonomy and control over their own lives and outcomes. The third research question of this study sought to further understand the types of outcomes emerging adults experienced when direct, indirect, and direct yet supportive parental expectations were conveyed.

\section{Emerging Adult Outcomes}

In the third research question, I sought to understand emerging adults' perceived outcomes of parental messages about expectations about college. Participants described three primary outcomes of parental messages about expectations in college that seemed to differ by parents' message types from research question one. First, emerging adults shared mental health, well-being, and academic motivation/success outcomes. Second, emerging adults talked about how their parents' messages impacted the degree to which their college experiences were positive or negative. The third outcome participants discussed was learning more about 
themselves through introspection. The following sections will detail these outcomes from parental messages about college.

\section{Mental Health, Well-Being, and Academic Motivation/Success}

All emerging adults revealed some mental health, well-being, and/or academic success/motivation outcomes due to parental messages about college. It was impossible to tease out the personal and academic outcomes in the following excerpts, because participants often interweaved them in their stories. It was clear that participants perceived that personal well-being and academic outcomes inform each another.

Indirect Supportive Messages, Mostly Positive Outcomes. When participants recalled supportive parental messages, most emerging adults, like Hillary, felt emotionally happy, sustained, and academically successful:

Definitely happy and fulfilled. Definitely. I think just kind of knowing that no matter what my parents support me and are supportive of where I'm at, and I think that's a huge, a huge boost for me and a huge comfort for me is knowing that they support me and that getting those kinds of messages. Sometimes they're just like exactly the kind of messages I need. If I've had a long day is sometimes just really good reminders of how far I've come and, and where I'm at right now, and then also just kind of making sure, again, that I'm taking care of myself.

Michael attributed his confident self-esteem, academic identity, and impactful collegiate experiences to the supportive messages he recalled receiving from his parents throughout childhood:

I think they made me confident. My parents have always been giving me messages of intelligent, smart and friendly, [Michael] is empathetic. And so that really informed my 
identity. As I became more independent, you know, if I'm in the classroom, I feel comfortable participating, I feel like I have something valuable to say, I feel like I bring a good perspective to the table. I feel like I can do well in each process. Regardless of the difficulty, like, I feel like I can approach classes with that kind of confidence. And then socially, kind of kind of the same way. Feeling like I was relating with people that I was good to, definitely shaped the way I approach social situations now.

Even when it came to academic criticism, some participants, like Chloe, emphasized how the supportive messages they received helped reframe their successes and struggles in college in a constructive way:

An instructor will be like, this is a really bad quality. And I will say, okay, and then I'll tell myself like, like, I did my best, you know? I never like, I'm not concerned about grades. I suppose I would be if my parents had expectations of them. I don't even check my grades. And I should, I think. So, their messages helped me to not internalize negative feedback. And it's also because they don't have really strict expectations. It's helped me to, like, accept feedback and be like, okay, like, I'm not perfect, but my parents didn't expect me to be perfect, so it's okay. If that makes sense.

Michael shared how supportive messages from his father helped him feel validated in his academic endeavors:

I went up to my dad and I said, wow Dad, you are relentless with this house, it's impressive. And my dad was like, well you're a relentless academic, you're always writing you're always reading. That's super impressive. And to me, that meant a lot ... for him to say, I recognize that you're doing hard work, you're doing mental labor and that meant a lot that that made me feel very validated. And I think he wanted to let me 
know that even though we might not agree on his version of like, what a good man or something is, or a man does look different from him validates and sees the work I put in. Although children often framed parents' supportive messages as tied to positive outcomes, some emerging adults, like Chloe, experienced mental and emotional stress because they felt confused and frustrated by a lack of clear expectations and guidance on how to be successful:

Sometimes I think that not my parents not realizing those expectations reinforced my thoughts about how, how high their expectations might be, if that makes sense. So like, kind of like confusing, because if I told you like, I want you to have a B average, which I know like some parents do, they tell their kids, then I'd be like, okay, that's my expectation. But my parents are like 'we want you to learn a lot. And we want you to do your best and want you to try hard and they want you to get the experience that we know you can have'. Okay, but what does that look like? So I think sometimes that's a little bit frustrating, maybe a little bit stressful, because it's like, okay, well, they're not telling me what they want, but I feel that they want me to have straight A's and get these specific awards, and have no student loan debt and go to grad school and you know, like, along those lines.

Chloe's unique experiences serve as an important reminder that while supportive parental messages may provide emerging adults with positive personal and academic outcomes in college, supportive messages may also leave emerging adults without adequate guidance for expected performance resulting in mental and academic stress. Participants who recalled indirect and supportive messages about expectations from their parents about college shared mostly 
positive outcomes on personal health and academic success. Academic and mental stress for these participants usually occurred due to a lack of clearly-stated expectations for college.

Directive Messages, Mostly Negative Outcomes. Conversely, the majority of students who recalled directive and highly controlling parental messages about expectations in college expressed severe strain, both mentally and academically, resulting from their parents' messages about expectations. Here participants felt stress to do well out of obligation to their parents' expectations and/or due to high parental overinvolvement/control. Participants like Brittany shared that there was a lot of pressure and stress to meet expectations:

A lot, a lot of pressure and stress. The first round that like, really just does not help. There's already so much pressure and stress involved in being in college. Yeah, it's just, it's another way of like, I'm not meeting their expectations. And I'm going to have to deal with the repercussions of that. And they're not going to be proud of me. I, yes, did not handle that pressure well, at all. As I mentioned, I've been in therapy for like three or four years now. I mean, I was, I was pretty much a wreck. Like when it got to the point where I realized that like I was not capable of meeting those expectations.

Brittany exemplified the immense strain that recalled, directive parental messages about expectations may impart. Elliot also shared surmounting outcomes of emotional and academic stress, and self-doubt, due to parental expectations, over-involvement, and control:

I think the expectation that you're so it's not an individual, maybe so much as it is a sort of openness for that information just ebbs and flows. More, there's just sort of nothing, nothing that you wouldn't tell each other or comment on or not know about each other that it would just be so sort of unnatural openness that would occur and I, I don't think that's normal in families. But I think that was their desired goal. And so that they tried to 
implement it. They're over involvement and they're over-controlling I think, in many ways, I think led to the opposite reaction of wanting to withdraw and wanting to keep some parts for myself ... It definitely made me feel deficient. And I think to some extent, still does, I'm still sort of working through those expectations that I think but knowing that I'll never have a family in the way that they want me to at least. Yeah, it makes me feel a little deficient.

Beyond feeling deficient and self-doubting, some participants, like Gloria, described the pressure of trying to live up to parental expectations in college:

My mom was so big on like me being a lawyer or, you know, those top paying jobs. Um, I struggled my first semester trying to live up to something that I didn't want, or something that I enjoyed doing, but not something I loved or wanted to do as a career. Because of my mom. My first semester was absolutely horrible. Because I was trying to do what they wanted me to do.

While doing what her parents wanted, Gloria described feeling burnt out and stressed. Frank also described feeling obligated to his parents out of a strong will to please and appease their expectations:

Definitely obligated because I felt like I was obligated to do really good work. But my parents were the ones that were funding my college. Because like, I felt like I had to submit good work. Otherwise, like, it wasn't worth it to them. And I felt like I, they deserved me to succeed if they were paying for it. So I was very upfront and you know, felt like I really needed to succeed in the classroom. I didn't necessarily feel lonely or ostracized or anything like that. But I definitely felt like I was. I owed it to my parents to 
be successful, because of the fact that they were paying for it and gave me all of these, you know, messages that indicated that I needed to be successful.

Some participants, like Isabel, even described how not living up to expectations would have led to being viewed as a disappointment:

I would say for the most part, they made me feel pressured is the word I want to use. Um, just because starting off, it was all that frequency of messages, and it was you need to go to school and it was very rigid and agenda based. So, it was like, I felt this pressure to either, like, go to school and uphold their expectations or not follow what they wanted to just kind of be a disappointment.

Beyond personal strain and self-doubt, many participants whose parents were direct in their messages also viewed teacher feedback/academic criticism as harsh and personal. For Isabel, she equated criticism from instructors to harsh parental messages about expectations in college:

I think that, yes, they've [parental messages about expectations] definitely impacted me. I think that I tend, I used to tend to take instructor feedback very harshly, very personally. So in my first few years of school, when I would receive feedback from instructors, um, it was very similar to receiving those messages from my parents. So I kind of associated those two with each other.

Some participants who felt distress from living up to their parents' expectations saw their parents' messages as motivating but not necessarily in a positive way. Isabel explained how their academic success was motivated by parental expectations, for better or worse: 
I think that early on, they helped motivate me. However, I don't really know if it's in a good way or a bad way, though, because they motivated me, but at the same time, the motivation was to uphold their expectations.

For some participants, like Brittany, academic success and motivation came initially from parental expectations, but ultimately, she developed her own set of expectations. She shared that if not for her decision to separate herself from her parents' expectations, she would not have attained academic success:

I have learned. I think, like, going through college, like doing it for my parents taught me what to avoid, if that makes sense. And now I'm able to do this college thing, $100 \%$ on my own terms, and I have all of the like, power in the situations, my parents, as I said, can only be involved in it so much as I let them. And had I not gone through the experiences that I went through my first time around, I may not have learned that lesson, and things would be very different, and not necessarily in a good way. Participants described how a lack of validation and parental disconnection from their emotional and physical well-being caused them to struggle even more. Nyesha, who talked about her parents' messages typically as directive but supportive, described the directive nature of some of the messages as straining to her mental health and self-esteem, but also lacked validation of her abilities and academic performance:

I definitely say that they impact my mental health and not in a good way. My dad can be very harsh, like ticked, yelling kind of in a way is quick to anger. So definitely makes me sometimes said I'm like, I'm not good enough. I need to be doing this. So I think it puts a lot of pressure on me personally as a person and my self esteem and I'm like, I could be doing better I could be doing this. Sometimes I think that it makes me overthink and be 
so critical of myself that when I accomplished something I am upset or like, I'm like, I could have done better. Oh, or I could have approved this instead of being happy about the accomplishment that I have made because, like, there's so much room for improvement or like oh, like I really like I screwed up on this I need to do better. So I have to say the lack of validation hasn't really been helpful for my mental health personally.

For Gloria, her parents' inability to recognize her academic efforts as worthwhile and meaningful caused her to struggle:

Because I feel like even if I do explain it [college as hard] to them, they're still seeing it as like, like that. It's this easy, obtainable thing. And I'm like, it's not undergrad guys. Like this is a whole new ballpark. Like, like I've had mental issues, mental issues come up. And that's when they'll like, shut me off. That's when the academic and all that gets shut off. Once it tapers into I'm having mental problems with school it's like, okay, we don't want to talk about this. So that's when the academic kind of stops and they don't want to hear anything, is if it goes towards I'm struggling. And my mental health is struggling so much because of this.

In this example, her parents only wanted to be overinvolved when it came to determining her academic decisions but did not want to know about any negative outcomes that stemmed from these high expectations. Like Gloria, Brittany described how a lack of attention to her personal and emotional needs led her to even heavier challenges with mental health:

Just felt like a lack of care. And I don't know, probably a word for that, that I'm not thinking of. But yeah, it was, it was the ignoring of the issues that I was facing and the struggles that I was facing at the time, and instead prioritizing my like, grades and like on 
paper successes. Yeah, it felt like they did not care about like me, personally. There was a lack of validation? In my personal emotional and mental well being? Until it got, really, really bad. Yeah.

Participants who recalled direct and controlling messages about expectations from their parents about college shared mostly negative outcomes on personal (mentally/overall) and academic stress. Participants felt strained to do well out of obligation to their parents' expectations and/or due to high parental overinvolvement/control. Without parental validation, participants expressed heightened struggles with personal and academic stress.

Direct and Supportive Messages, Positive Outcomes. Finally, participants who reported direct but supportive messages seemed to experience positive mental health and academic success outcomes. For instance, Dejah, who tended to recall messages that were directive but supportive, framed her parents' messages and their influences on her as helpful for establishing a more realistic outlook on college and allowing her to build autonomy:

I've heard from a lot of people that they think my parents are strict, or controlling ... But really, it's just that they care. And at the end of the day, they just want to know that I'm satisfied, and happy with the choices I've made. I think they've definitely helped me be successful in the classroom as well as in like, my personal life. Because it just kind of was that ingrained, explicit attitude of, you know, we are successful, we do things we have, you know, we have the capability to do great things. And just kind of that belief and expectations that, you know, we can do good things we can apply ourselves, and all those kinds of things really hit home for me and made me realize that, like, I'm capable of, you know, being successful academically or, you know, really going the distance with other areas and just being successful overall no matter how hard those messages were at 
first. And, I think they've actually made me set goals that are more realistic, because like, before I was, I was more on the edge or on the verge of, you know, oh, I can do kind of whatever I want, like, everything's going to be an A all the time. But then like, you know, talking with my parents, it became more of, you know, you're not going to get an A on everything ... So I think it just made me realize that things are a lot more realistic. And, you know, you can't expect to do perfect on every single assignment, you just have to strive to be the best that you can.

Nyesha, who also recalled direct and autonomy-supporting messages, also shared how she preferred these messages over a lack of parental expectations even if they were not communicated in the most loving way because they led to realistic goals:

I rather have parents who are happy and who are involved in like wanting to know like what I'm doing instead of not caring whatsoever ... I think I would just say that overall I'm so glad that my parents have been here every step of the way, even though, maybe it's a cultural thing, it's been harsh or hard to listen to them, and I'm like oh my goodness I just can't deal with all of it. I'm glad that they do show some sort of effort and that they do care about me and want me to do my absolute best no matter what form of communication I'm getting from them because in the end, it just helps me have more realistic goals and shows how much that they do truly care about me even if it's not in the firmware love that I wanted.

All participants reported outcomes, positive and negative, regarding mental health, overall well-being, and academic success/motivation. Participants who recalled parents indirect and supportive parental messages typically experienced fewer strains on mental health and reported more positive and fulfilling academic success/motivation experiences. Participants who 
recalled direct and controlling parental messages tended to experience intense, negative strain on mental health, overall well-being, and academic success/motivation. Participants often discussed the importance of parent validation on self-esteem; their responses to academic criticism; and academic identity, success, and motivation. In the following section, I outline other summative emerging adult college experiences as influenced by parental messages about expectations about college.

\section{Summative College Experiences}

Participants also discussed outcomes of their parental message that are best labeled as "summative college experiences," as they touch on various components of their education that they believed parents' messages influenced, specifically their choices in college experiences and their feelings of loneliness or connection with others.

Choices in College Experiences. Most participants talked about how their parents’ messages about college often influenced their choices beyond but related to academics. For instance, children often internalized messages about college when deciding whether to participate in and prioritize activities outside of the classroom. For example, while Chloe emphasized how fulfilling her collegiate experience was, Isabel emphasized her regret in sacrificed social experiences, both because of parental messages/communication about expectations in college. For Chloe, supportive parental messages from research question one helped her to have a "fulfilling college experience:"

I think because my parents are so supportive, it's helped me to really have a fulfilling college experience. Like I was able to try a bunch of stuff, and balance working hard in school, and like having a social life and being involved extracurriculars, volunteering a lot, just stuff that I was really interested in. And so they've kind of pushed me to explore 
opportunities that I'm passionate about, or things they might be passionate about. And then they've been really supportive of all those decisions that I've made. So it like, college is very stressful. But it hasn't been made any more stressful by communication from my parents about it.

Conversely, Isabel recalled direct parental messages from research question one that emphasized getting good grades and success above social experiences:

Yes. I think that that looking back, I think about that a lot. That is probably one of my biggest like, regrets going through my undergraduate program was that I sacrificed a lot of social opportunities, because I wanted to uphold their expectations based on what they had communicated to me in all those frequent messages early on. So, I was more concerned with getting good grades and going through a program that they approved of and that we had talked about, then branching out and meeting new friends and doing fun things most of the time.

Beyond social experiences, Elliot attributed his sacrificed collegiate experiences (in his instance, building his own ideas) to the recalled, directive and controlling messages he received: College is about free thinking and genuine evaluation of ideas. And so I think I missed out on an earlier opportunity to do that in my undergrad. Now, now I don't talk to my parents about, like, the substantive parts of education, but, but yeah, I think I missed out on building my own ideas, early on, I guess, because I went in with the mindset of 'conserve the ideas that you have and the ideals that you have'.

These findings emphasize the implications of variant parent messages about expectations on the emerging adult's collegiate experience. Participants most often expressed that their social and personal experiences in college were fulfilling and exemplary when they had parents who 
they described as supportive. Participants who framed parents' messages as direct and/or controlling (from RQ1) expressed a sort of detriment and sacrifice to their social and personal experiences in college.

Loneliness and Connection. When participants perceived that their messages from parents about college were quite different from those others received, they felt lonely and disconnected. For example, as Nyesha talked about her collegiate experiences and the messages she received from her parents, she quickly came to realize how different she was from her friends:

Um, sometimes I do feel alone. When I get a certain message from family or like a certain thing that they told me that I need to do or like you're not doing this well enough. And I relay these messages to friends who have like, no clue what I'm talking about, like, oh, my goodness, like my parents never say that. Or like, they're like, I don't experience what you're talking about. So I do think it makes me feel sad when I'm like, why can't really relate to anyone, myself. And I do feel alone because I'm like, no one else is experiencing this. Or nobody else feels the same way that I feel.

Nyesha also shared how these differences soon became isolating and lonely: I've had friends who like just don't understand it. And I'm like, well, I just can't like [hang/go out, socially], you know? I said, like, I'm going home or like, I'm like, well, like, I have a curfew to get home. And my friends would say, like, why not? You're like 21? Or you're like, 20? I'm like, yeah, but it's like, I still have to follow rules. So I do think that some friends look at me differently. And they're like, oh, like we're not gonna even asked her to hang out. Or we're not even going to ask, because she can't do it anyways. 
Conversely, Brittany described how she connected with others through their shared experiences of directive parental messages:

I was lucky in that a lot of my friends were kind of in similar boats. And that, was that, everyone was like, former gifted kids, like really good in high school, and like, had a bit of shell shock when they got to college. So, I, nobody could really ostracize me because, I, we were all going through the same thing.

These findings on emerging adult collegiate outcomes on experiences with loneliness and connection illuminate the resonating salience of parental messages about expectations in college for the emerging adult and the continued implications on not only mental health and overall wellbeing but also relationship-building during college. These findings collectively emphasize the differences in emerging adult experiential outcomes when the messages communicated by their parents differ.

Perhaps the most fascinating emerging outcome of this study was that regardless of recalled messages, responses, and positive and negative outcomes on emerging adult college experiences, all participants talked about reflecting on their parental messages as a way to grow as a young adult. Here, despite all positive and negative outcomes of parental messages on these college students' experiences, emerging adults collectively viewed the parental messages they received about expectations in college as good faithed and sourced from a place of concern, care, and love.

\section{Reflection on Messages}

As participants reflected on their responses to, and experiences with, parental messages, they collectively seemed to construe these messages as positively impactful on their emergent outcomes in college and their acquired autonomy. Here, despite negativity and turbulence to 
emerge in some instances, most participants described their emergence as successful and the parental messages they received as good faithed, even if parents' messages were unjustified or painful still. Michael reflected on his experiences with messages about expectations he received from his mother as something that was more secure and logical towards helping him become successful and confident:

It was trying to be helpful. But at the time, it was hard to take because I knew that she was expecting such a higher level from me than my college. So I guess emotionally it felt overbearing, but logically, super useful. If that makes any sense ... I like, it no longer hurts me more like it's over time, and especially as I've grown in confidence, that that part of our relationship got better for me emotionally because it was more secure.

Brittany shared how her emergence into successful autonomy occurred despite her parents' expectations; but, how introspectively she owes the realization and success to being a "victim" of her parents' expectations:

Um, overall, I don't know if this is helpful, but I, I feel more and more strongly about how ridiculous it is that people are expected to go to college at 17 to 18 years old. I feel comfort in knowing that like, there's a statistic about how many people change their major however many times before they actually end up graduating. And it's kind of nice to know that like, nobody really knows what they're doing. When they go in, freshman year. I don't know. It's a slob system. And we're all kind of victims of it [parental expectations] one way or another and to just do what is best for you is always going to be best for you.

Elliot recognizes the same negative implications of the messages he received, but views those messages as hurtful then, but constructive and sourced from a place of love now: 
They have those desires for us because they want good things for us. But I also acknowledged that it was overly controlling and felt that way. I think it's in part due to the time of my upbringing. I'm gonna call it brainwashing now. But I think I thought that was, that was okay. And good.

In her experiences with direct and supportive parent messages, Dejah acknowledged that if it were not for her and her parents' successful re-negotiation of their relationship, she would not have emerged as successful and autonomous:

And so in the beginning, I felt like they were trying to push me into a specific avenue, lane, path for my life. And then as I got older, and I volunteered information of, hey, I did this in class. This was what I was super into. Now, I want to be a producer of radio, hey, I did this in class, and I want to be a producer of television. As I've changed my mind, because I've had more experiences, they've been like, oh, that's cool. So as I've gotten older, as I've gotten more assertive, as we've all gotten more comfortable with me being an adult and having freedom and I learned more. I don't really feel their expectations in that way ... Because even if I don't agree with what my parents have said, or how they say said it, I do know it comes from a place of love. I do know it comes from them knowing a little bit more about the world than me. And so I use it as a motivation, of just wanting to make my parents proud. Um, and then even if I don't follow the advice, they give me the suggestions they give me, or I don't come away from a particular conversation, feeling uplifted. I just take the sum of every conversation and just thank my parents just want me to be as successful as I can be. It's not what would make my parents happy. But it's what's going to put me at the best advantage in life. What's going to keep me sane, what's going to help me because I know that's all they want for me ... And so I 
think the biggest thing I've learned from thinking about what my parents want from me from college, and then having this conversation is our openness and willingness, willingness to have a conversation and dialogue has been key, because I'm sure they've gotten frustrated with me. And I definitely know that I've gotten frustrated with them. But yeah, we've each just had to relearn each other. They had to relearn me as an adult. I had to relearn who they are as an adult, and then value each other's experiences, their journey with academia, and then my journey as well.

These findings suggest that while the messages parents share about expectations with their emerging adult children may be salient and have a large influence on college student outcomes, the resounding implications of the emerging adult's ability to attain autonomy and emerge successfully may not be as drastic. Upon reflection, many participants described some level of gratitude toward their parents' messaging, as these messages influenced their ability to develop autonomy. However, the implications of diverse parent messages about expectations communicated throughout childhood and into emerging adulthood remain salient.

Research question three sought to understand the various outcomes emerging adults experienced in college because of parental messages about expectations. First, all emerging adults experienced outcomes on mental health, well-being, and academic success/motivation. Second, emerging adults described making particular choices in college experiences as a result of parental messages about expectations. Some emerging adults experienced loneliness or connection to others, based on how similar or divergent their parents' messages were compared to messages that their peers received. Third, all emerging adults introspectively described their autonomous emergence into adulthood as in gratitude towards, or despite, parental messages about expectations in college. 


\section{Conclusion}

In response to $\mathrm{RQ}_{1}$ participants reported three primary types of recalled parental messages about expectations about college: direct and prescriptive messages about college, indirect and autonomy-supporting messages about college, and direct but autonomy-supporting messages about college. For $\mathrm{RQ}_{2}$ participants reported utilizing three main response behaviors to parent messages about expectations about college: compliant and avoidant responses, autonomous responses, and autonomous and selectively compliant responses. Lastly, for $\mathrm{RQ}_{3}$ participants reported three main outcomes to parental messages about expectations in college: mental health, overall well-being, and academic motivation/success, summative college experiences (choices in college experiences, and loneliness and connection), and reflection on college messages.

Memorable messages framework was utilized to help make sense of why and how emerging adults recall, respond to, and become influenced by parental messages about expectations about college. 


\section{CHAPTER V: DISCUSSION}

The purpose of this study was to understand what memorable messages about expectations for college that emerging adults could recall having received (or still receive) from their parents, how they responded, and how they perceived their college experiences to be influenced by these messages. Emerging adults recalled three different types of parental messages about expectations, responded with three different response behaviors, and described three different outcomes on their college career. I used Knapp et al.'s (1981) memorable messages framework to understand what memorable messages emerging adults could recall having received from their parents and why they were held salient. In the following sections, this study's findings will be summarized and followed by the implications and limitations of the study, with directions for future research.

\section{Summary of Findings}

From the in-depth, qualitative interviews, I coded the transcript data for emergent themes until the points of redundancy and saturation. Themes were then categorized along responses to research questions posed. Once categorized, emergent themes were narrowed for each research question posed. Although findings in the results section were divided by their pertinent research question, there was an obvious and deep intertwining of themes. Participants recalled memorable messages that were significant and long-lasting and that influenced their decisions about and in college, their responses, and the outcomes associated with the messages. In the following section, I summarize the findings of this study organized by research questions posed.

In the first research question, I sought to understand the memorable messages emergent adults recall having received, or still receive, from their parents about expectations about college. Participants recalled receiving three types of messages from their parents about expectations 
about college. First, participants recalled parental messages that were stated outright, as participants perceived as part of a largely agenda-based, directive conversation seeking to convey explicit expectations. Second, participants recalled parental messages that were more indirect and provided support to the child. Here, messages about college were not stated as outright expectations, but more as a part of larger, continuous conversations that sought to merely invoke thoughts about college and the emerging adult's future. Third, participants recalled messages that were direct yet still provided support and aimed to create the child's autonomy and self-efficacy.

In the second research question, I sought to understand how children responded to their parents' messages about expectations about college. Participants recalled responding three ways that often differed based on parents' message types from research question one. First, children whose parents were direct and prescriptive tended to respond through compliance or avoidance. Second, participants whose parents were indirect and supportive tended to use autonomous responses. Third, participants who received direct, yet supportive messages responded with autonomy and selective compliance.

In the third research question, I sought to understand emerging adults' perceived outcomes of parental messages about expectations about college. Participants described three primary outcomes of parental messages about expectations in college that sometimes differed by parents' message types from research question one. First, emerging adults shared mental health, well-being, and academic motivation/success outcomes. Second, emerging adults talked about how their parents' messages impacted the degree to which their college experiences were positive or negative. The third outcome participants discussed was learning more about 
themselves through introspection. These summative findings allude to important implications of this study.

\section{Implications}

Due to this study's nature in examining the parent-child relationship and parental messages about expectations shared throughout development, this research has resulted in various implications. Throughout all 14 participant interviews, emerging adult college students could recall messages about expectations that they had received (or still receive) from their parents about college, how they responded, and what their perceived outcomes of these messages were in college.

\section{Messages}

It is important to study emerging adults in college, and their relationships with their parents, because the way that they are raised can have important implications on their college experiences. While Knapp et al.'s (1981) memorable messages framework might suggest that specific messages (i.e., the exact content shared during interactions) will be recalled and held salient, this research found that perhaps emerging adults will not always recall specific messages word-for-word but instead types or themes of messages that occurred repeatedly. Indeed, the types of messages shared throughout development from parent to the child may have resounding impacts on the parent-child relationship (Cheung et al., 2019). Parents may knowingly or unknowingly instill unrealistic perceptions of their expectations by stressing the importance of education, success, occupational outcomes, and responsible social behavior to the detriment of the emerging adults' personal development (Agliata \& Renk, 2009). The nature of the emerging adult's transition into adulthood does require a certain maintenance for the parents as "authority figures" throughout the college experience (Archer \& Lamnin, 1985; Wang \& Heppner, 2002); 
however it seemed that in many instances, particularly when giving more heavy-handed messages about college, participants perceived that parents were overinvolved (Rudy et al., 2014) . Accordingly, this research suggests that the types of message shared during emerging adulthood may be highly recollective in nature and have lasting impacts due to the importance the emerging adult places on the parent-child relationship and how much the children care about what the parents think they should do.

This research also emphasizes the influence of family norms and culture. College students' perceptions of their recalled parental message types about expectations and their collegiate experiences sometimes varied based on culturally-based emphasis of expectations and parental control messages/behaviors. This research found that, indeed, cross-culturally, children approach tasks based on the influence of their parents' culturally-based beliefs (Jose \& Bellamy, 2012). These findings suggest that taking cultural variance into account, highly imposed expectations (in this study, academic expectations) tends to be connected to children's greater self-doubt and self-critical evaluations of their own performance and decisions. For individuals who identify within the Asian culture, for instance, high value is placed upon intelligence and even higher expectations on academic excellence/success than most other cultural groups (Naumann et al., 2012). Accordingly, participants like Mrudita (an Asian-Indian) expressed consistent academic stress and self-doubt due to highly imposed expectations for higher intelligence and great academic success. Indeed, parenting norms in certain cultural contexts pave the way for negative parental reactions to violated expectations (Rudy et al., 2014). In this study, when expectations were violated, some participants described their parents using different types of psychological control tactics (i.e., blackmail, guilting) to try and persuade their children to comply with expectations again (Rudy et al, 2014). Certain cultural norms, history, and 
traditional family practices for a child's upbringing may impose higher expectations upon those emerging adults who were raised within their cultural values while living in America (Vitoroulis et al., 2012). For individuals within the Hispanic culture in this study's sample, history and family traditions/norms became particularly salient when it came to educational attainment. For these individuals, academic attainment in America was the largest expectation emphasized due to the inability for ancestral family members to have attained the same. For individuals within the Black/African American culture/community, historical roots and shared experiences became (rightfully) extremely influential on the types of expectations parents communicated to their children seeking to combat extreme systemic racism and false assumptions. For instance, Dejah expressed how being a Black woman in a marginalizing and highly stigmatic American society really influenced her parents' expectations that emphasized earning a degree and pushing through college "no matter what".

Various communicative patterns (and resulting messages about expectations) between parents and children can either hinder or strengthen the child's ability to emerge as autonomous (Gridley et al., 2018). Accordingly, this study's findings echoed Gridley et al.'s (2018) analysis that parental communication that was characterized by consistent care, compatible behavior, and open communication correlated with adults recalling messages about expectations about college that were perceived as indirect and autonomy-supporting. Similarly, parental communication that was perceived as demeaning or dictating, led emerging adults to recall parental messages that were direct, prescriptive, and undesirable overall (Gridley et al., 2018; McKinney \& Kwan, 2018). In the third emergent outcome, direct yet open parental communication correlated with adults recalling messages that were harsh at first, but helpful overall. Indeed, recalled messages about parental expectations about college by emerging adults either varied by parental 
manifestations of involvement/overinvolvement and control. When manifestations began to "tip" towards overinvolvement and control, participants typically recalled messages that were directive and prescriptive (Faherty et al., 2020). Additionally, the types of memorable messages that children could recall created a context for family communication (particularly how emerging adults responded to these messages) into emerging adulthood (Michalos et al., 2014). When parental messages about expectations were indirect and autonomy-supporting, participants described being able to communicate their own opinions and share their decisions with their parents. When parental messages about expectations were direct, prescriptive, and controlling, participants described feeling unable to communicate opinions and never able to share decisions without receiving more negative and harsh parental messages about expectations, until finally fighting back.

Messages about parental expectations do alter emerging adult experiences (Dorance Hall \& Scharp, 2018). Emerging adults' ability to acquire autonomy and self-identity can only be afforded by parent messages that allow for autonomy and independence (Faherty et al., 2020; Ryan \& Deci, 2000). In such cases, emerging adults responded autonomously. However, my findings suggest that in some instances, when too much support and autonomy is provided without any expectations, emerging adults may become frustrated without direct guidance. When emerging adults recalled parental messages that directly enforced expectations and inhibited personal development (e.g., blackmail, obligation, guilting, aggression), they typically responded with messages that were compliant and/or avoidant (Infante et al., 1994; Planalp, 2000; Tao \& Hong, 2004). In these cases, emerging adults sought to avoid further conflict and negative messages with and from their direct and prescriptive parents until some sought also to respond with intentional violation of parental expectations for their own autonomy. Ultimately, all 
emerging adults whose parents shared direct messages emerged autonomously on their own terms following some familial turbulence. Fascinatingly, direct and autonomy-supporting responses afforded emerging adults the most constructive ability to take some directive parental messages into consideration while also acquiring autonomy. Here, parent messages allowed for autonomy and independence, but still enforced some sense of realistic and foundational selfexpectations. The direct and autonomy-supporting parental messages about expectations was unanimously construed by participants as constructive, foundational, and helpful.

\section{Parental Involvement versus Overinvolvement}

Participants shared the differences between parental involvement versus overinvolvement. Indeed, as emerging adulthood continues to be a stage of life wherein parental involvement is necessary (Arnett, 2004), parental overinvolvement and control may soon become normalized to the emerging adult's detriment. Participants emphasized the adverse effects of overinvolvement on the parent-child relationship and resulting messages. At the collegiate level, parents' indirect and supportive messages about expectations about college may allow children to develop autonomy and have positive college outcomes (Arnett, 2004). Overinvolvement and control within the parent-college student relationship exists where parental desires to be enmeshed with their children, through an overemphasis of direct and prescriptive messages about expectations (Agliata \& Renk, 2007) and may soon lead to a plethora of negative implications on emerging adult outcomes (Hesse et al., 2018; Lynne et al., 2017). For example, participants whose parents may be defined as "helicopter parents" who practiced excessive involvement and the use of inappropriate tactics, stripped college students of their ability to establish autonomy (Hesse et al., 2018) until the emerging adults decided to violate these expectations. Similarly, participants who perceived that their parents desired to be enmeshed 
(i.e., enacting high behavioral and psychological control; Kaniusonyte \& Laursen, 2021), and whose parents typically desired conformity over conversation (Ritchie \& Fitzpatrick, 1990), described experiences with high amounts of identity crises, mental health detriments, and academic strain. These findings emphasize the positive and negative implications on emerging adult outcomes that are important to consider.

\section{Outcomes of Parental College Messages}

All emerging adults in this study experienced some mental and academic stress regardless of recalled parental messages, but also sometimes as a direct result of parental messages about expectations. For the emerging adults in this study, conflict between the actual self (i.e., attributes that individuals possess currently), and parental expectations for the ought self (i.e., attributes that the individuals believe they should or are obligated to possess), as well as the ideal self (i.e., attributes about the self the individual wishes to possess), and parental expectations for the ought self (Higgins 1987), did concurrently cause the college student to experience certain positive and negative perceptions of their overall college experiences and academic success and achievement (Higgens et al., 1986). For these participants, while indirect and autonomysupporting messages often afforded autonomy, self-identity, and motivation/success; in some cases, it also failed to clearly communicate successful expectations and outcomes. However, wherein direct and prescriptive messages inhibited autonomy and were perceived as controlling, expectations became overemphasized, obligatory, and often strained participants between "having to" and "wanting to" succeed (Wang \& Heppner, 2002). These collective tensions resulted in academic and personal stress but was alleviated when parents shared messages of support, validation, and connection to their emerging adult's emotional and physical well-being. 
Overall, emerging adults feel more validated and are academically motivated and successful in situations in which they experience academic and emotional support and consideration and care is allotted through supportive and open messages (Tang et al., 2018) and when responses to criticism are construed as constructive instead of combative. To foster emerging adults' positive academic and personal identities, these findings suggest that careful attention must be given to how parents convey support and guidance throughout childhood and emerging adulthood that allows for development of autonomy. To truly support emerging adults in this manner, parents must begin by examining their parenting type/style, and critically examine messages they communicate to children. Parental messages about expectations in college may have salience and resonating value and both positive and negative implications on collegiate experiences throughout every formative life stage.

Other positive and negative outcomes on emerging adults in college emphasized the varying impacts of different types of parental messages about expectations about college. Some emerging adults discussed the inflicting nature of directive parental messages on their ability to fully enjoy their college experience, while others attributed their fulfilling experiences to the supportive messages they received. Emerging adults described how direct and prescriptive messages led them to feel lonely because of their unique experiences, while others described feeling connected to college peers whose parents communicated similar messages. When children's parental goals and personal goals aligned, student persistence was, indeed, further facilitated (Wang \& Nuru, 2017). These findings illuminate the potential for the emerging adult (particularly those who received and internalized prescriptive and controlling messages) to still pursue their own autonomy and persist in this manner, although the factors that lead to their ability to develop autonomy was not uncovered in these data. 
Ultimately, some participants perceived parents' expectations as harsh, and while many emerging adults saw these expectations as unfair, they worked through them in different ways (i.e. compliance, avoidance, turmoil). Importantly, upon reflection, all participants came to the same conclusions that parents cared and wanted the best for them. In the end, all emerging adults acquired their own sense of autonomy. Despite the questionable, demanding, overinvolved, and controlling nature of direct and prescriptive parental messages, the messages may serve as a positive source of perseverance in the emerging adult upon reflection, representing the dark (stressful) and light (perseverance) sided nature of direct and prescriptive parental messages about expectations.

\section{Limitations}

The findings in this study must be interpreted in light of some limitations. First, while this was a relatively diverse sample, and considering I recruited from a medium-sized midwestern campus, a larger more diverse sample could have resulted in a more nuanced understanding of emerging adults' recollection, response, and outcomes of parental messages about expectations about college. Of the 14 participants, 9 were female, 9 were white, and 12 were under the age of 25 . Even though the point of this study was to better understand individual experiences and not generalize, a sample of differing ethnicities could make for a better, more representative collection of findings.

The second limitation is regarding the unique circumstances of the COVID-19 pandemic. At the university in which I conducted this research, remote learning was enacted to ensure student safety. Because of this, and CDC safety regulations, I was decided not to conduct inperson interviews for my safety and my participants' safety. As a result, I was unable to see some participants in a "live" format (aside from a few who opted towards video conferencing). 
Without nonverbal feedback, my readings and understanding of participants' realities could be altered. Third, although my interview protocol covered a wide depth of content and elicited robust responses, my protocol could have placed a larger focus on specific messages participants could recall receiving from their parents about expectations about college. A final limitation was the bias that accompanies one-on-one interviews and individual research. People want to display themselves in the best way possible and therefore may not want to share thoughts or feelings with interviewers that place them in a negative light. For the purpose of gaining a deeper, qualitative understanding of participants' experiences, free of bias, researcher triangulation would have been preferred to produce more verifiable data readings and analyses. However, having personally experienced parental messages about expectations regarding college, as the researcher, perhaps being closer to the research/data through shared experiences afforded me a higher ability to address reflexivity and awareness and accordingly avoid bias. In the final section of this study, I illuminate the value of the research conducted to inform future studies.

\section{Future Directions}

This study was a first step towards a better understanding of the experiences emerging adults may have with recalled messages about parental expectations regarding college. While these findings suggest that direct and prescriptive messages about parental expectations may espouse harsher outcomes on the emerging adult but still ultimately lead to their autonomy through turbulence, there may still be research to be conducted that examines the parent-child relationship post college, post parental messages about expectations, and post collegiate outcomes. Such a study may be conducted using Baxter and Bullis' (1986) relational turning points framework that may help to explain a change in the parent-child relationship due to factors (messages) that influenced that nature of interactions between parent and child. 
Additionally, this research provides a pathway for future research that seeks to examine the role of emerging adult's self-reflexivity within the parent-child relationship.

As suggested by Faherty et al. (2020), emerging adults at this stage felt some inclining of obligation to maintain relational ties with their parents as they continued to seek parental support, guidance, and advice while they emerged into adulthood. Participants struggled with the degree to which they wanted to maintain strong ties with parents after they started college. Many concluded that they were obligated to maintain relationships with parents but desired a renegotiation of boundaries that often did not occur. Participants shared that the messages they could recall from their parents included some sort of expectation to maintain communication and the relationship during college. As Chloe recalled:

I think they want to hear from me. And I think just from like a, like a family perspective, like we're close family. And so like, I want to be close to my parents, and they know they want to be close with me. So I feel obligated to like check in with my dad, because like I said, like, he doesn't usually text me first, you know, but sometimes they'll text me be like, hey, hope you're having a great day. And I also, sometimes I'll think, like, oh, I haven't called my parents in a couple weeks, like I should call them. Or sometimes they'll say what is the next time you're coming home? So it's clear in our conversations that they want to be close with me even when I'm not at home. So I feel an obligation to continue that relationship and make sure that I sustain the level of closeness that I'm used to with them, even when I'm not home a lot, or even when I'm not living with them.

While Chloe emphasized the relational norm of familial closeness as her source of obligation to willingly maintain the relationship, Elliot emphasized the need to maintain ties, even when unwanted, because his parents needed support and he was obligated to care for them: 
And so, yeah, but I do it anyway. Because I think, God, like, they're old. And they have no friends, you know, like, like, like most old people. And like, we're their friends. And they need us, you know. And it just makes them so happy ... It feels like an obligation. Chloe and Elliot's talk emphasized the unique circumstances of renegotiating the parent-child relationship, and its maintenance, while in college. Thus, future research should seek to examine how emerging adults maintain both wanted and unwanted relationships with their parents in college, and how they renegotiate their roles and relationships with family.

Expectancy violations theory provides viable direction towards understanding how and why parents might react when expectations are violated, especially in the context of the parentchild relationship. Prior research suggests that established parent-child relationship creates a context ripe for relation-specific expectations to be created based on knowledge of the other and relational history (Miller-Ott \& Kelly, 2015). Within research question one, participants often expressed that parental expectations were more highly construed from prior experience, strongly held familial beliefs, and the overall relational history between parent and child/emerging adult. While these expectations may then be directly or indirectly communicated within parental messages, the strength of expectations held in certain contexts may lead to higher valence judgements when expectations are violated. As a result, the emerging adult may feel higher amounts of pressure and/or stress to achieve parental expectations and may enact compliant/avoidant responses to avoid negative, combative parental responses to violated expectations until reaching the point of desired resistance and autonomy. Conversely, in some interviews, participants described their responses to parental messages as autonomous and selective of their own desired outcomes with abundant self-efficacy. Overall, future research examining parental expectations (their formation and valence judgements by parents) may help 
illuminate how expectations are created, how they are communicated, and why they are held as salient by parents.

Throughout the interviews, some participants talked about earning their college degree "for them" (their parents). In most cases, such a desire was sourced from cultural expectations and/or unique familial, cultural experiences. Some emerging adults even expressed how it was their job to become successful in order to care for their family in the present and future. Such findings suggest future research that seeks to understand specific, culturally-influenced expectations communicated from parents to their children on emerging adult collegiate outcomes. Here also, it would be important to research the differences of individualistic versus collectivistic cultures on the types of expectations held. Certain cultural norms, history, and traditional family practices for a child's upbringing may impose higher expectations upon those emerging adults who were raised within their cultural values while living in America (Vitoroulis et al., 2012). The cross-cultural tensions presented in this provide heuristic value to researchers who may aim to study sojourner students in America and the culture specific expectations that may impart cross-cultural strain.

Based on the positive implications of supportive parental messages of validation and connection to their emerging adults emotional and physical well-being during academic stress, and the positive implications of supportive messages for academic success and motivation, this research also provides a pathway for future research that seeks to examine the impacts of teacher validation on emerging adults' college experiences and how the teacher-emerging adult relationship may help foster positive academic identities. In such research, it may also be fascinating to study the additional impacts of teacher validation/motivation and support when emerging adults receive directive messages about expectations about college from their parents. 
Finally, this research provides a unique pathway towards understanding the differences in college experiences emerging adults may encounter as a result of parental messages about expectations about college. Future research looking into new parent messages about varying expectations (e.g., body image, health, sibling comparisons), and their impact on emerging adult college and identity/adulthood experiences and outcomes, may be largely impactful to uncover. The parent-child relationship, and messages shared, is a unique context ripe for communication research that seeks to examine the outcomes of parental messages about expectations on emerging adults.

\section{Conclusion}

In this study, I established that parents' expectations about college may have harsh and resounding implications on an emerging adult's ability to transition into adulthood with a positive sense of identity and autonomy. Additionally, these messages have both positive and negative influences on their relationships with parents, mental health, and college experiences. In the end, all emerging adults acquired their own sense of autonomy. However, direct, prescriptive, and controlling parental messages about expectations presented more negative and hurtful outcomes on emerging adults than did indirect and autonomy-supporting, and even direct and autonomy-supporting messages. Thus, even if parental messages about expectations may be coming from a place of love and concern, they have enduring implications on the emerging adult. This study illuminates the importance of studying the family communication that takes place between parents and children, and certain parental messages that may impart an innate, sometimes detrimental, desire to fulfill parental expectations. 


\section{REFERENCES}

Agliata, A. K., \& Renk, K. (2007). College students' adjustment: The role of parent-college student expectation discrepancies and communication reciprocity. Journal of Youth and Adolescence, 37, 967-982. https://doi.org/10.1007/s10964-007-9200-8

Agliata, A. K., \& Renk, K. (2009). College students' affective distress: The role of expectation discrepancies and communication. Journal of Child and Family Studies, 18, 396-411. https://doi.org/10.1007/s10826-008-9244-8

Akhtar, Z., \& Aziz, S. (2011, June). The effect of peer and parent pressure on the academic achievement of university students. Language in India, 11(6), 254-265. https://languageinIndia.com

Aldeis, D., \& Afifi, T. D. (2013). College students' willingness to reveal risky behaviors: The influence of relationship and message type. Journal of Family Communication, 13(2), 92113. https://doi.org/10.1080/15267431.2013.768246

Alford, A. M., \& Harrigan, M.M. (2019). Role expectations and role evaluations in daughtering: Constructing the good daughter. Journal of Family Communication, 19(4), 348-361. https://doi.org/10.1080/15267431.2019.1643352

Arnett, J. J. (2004). Emerging adulthood: The winding road from late teens through the twenties. Oxford University Press.

Association, A. P. (2020, January). Parenting Styles. https://www.apa.org/act/resources/factsheets/parenting-styles

Aziz, S., Hassan, H., \& Atta, N. (2011, June). Role of parental involvement in their children's studies and children's achievement. Language in India, 11(6), 158-163. https://languageinIndia.com 
Basar, U. (2018). Studying for one's self vs. studying for (significant) others: A critical review of the notion of academic motivation in Asia. Global Media Journal: Turkish Edition, 9(17), 1-17. https://www.globalmediajournal.com/

Baumrind, D. (1971). Current patterns of parental authority. Developmental Psychology, 4, 1103. https://doi.org/10.1037/h0030372

Baumrind, D. (1991). The influence of parenting style on adolescent competence and substance use. The Journal of Early Adolescence, 11, 56-95. https://doi.org/10.1177/0272431691111004

Barge, J. K., \& Loges, W. E. (2003). Parent, student, and teacher perceptions of parental involvement. Journal of Applied Communication Research, 31(2), 140-163. https://doi.org/10.1080/0090988032000064597

Barton, A. L., \& Hirsch, J. K. (2016). Permissive parenting and mental health in college students: Mediating effects of academic entitlement. Journal of American College Health, 64(1), 1-8. https://doi.org/10.1080/07448481.2015.1060597

Baxter, L. A., \& Bullis, C. (1986). Turning points in developing romantic relationships. Human Communication Research, 12, 469-493. https://doi.org/10.1111/j.14682958.1986.tb00088.x

Braun, V., \& Clarke, V. (2006). Using thematic analysis in psychology. Qualitative Research in Psychology, 3, 77-101. https://doi.org/10.1191/1478088706qp063oa

Castro, M., Exposito-Casas, E., Lopez-Martin, E., Lizasoain, L., Navarro-Asencio, E., \& Gaviria, J. L. (2015). Parental involvement on student academic achievement: A metaanalysis. Educational Research Review, 14, 33-46.

https://doi.org/10.1016/j.edurev.2015.01.002 
Chen, W., \& Ho, H. (2012). The relation between perceived parental involvement and academic achievement: The roles of Taiwanese students' academic beliefs and filial piety. International Journal of Psychology, 47(4), 315-325. https://doi.org/10.1080/00207594.2011.630004

Cheung, R. Y. M., Leung, M. C., Chiu, H. T., Kwan, J. L. Y., Yee, L. T. S., \& Hou, W. K. (2019). Family functioning and psychological outcomes in emerging adulthood: Savoring positive experiences as a mediating mechanism. Journal of Social and Personal Relationships, 36(9), 2693-2713. https://doi.org/10.1177/0265407518798499

Cionea, I. A., Van Gilder, B. J., Hoelscher, C. S., \& Anagondahalli, D. (2018, November). A cross-cultural comparison of expectations in romantic relationships: India and The United States. Journal of International and Intercultural Communication 12(4), 289-307. https://doi.org/10.1080/17513057.2018.1542019

Cushman, S., \& West, R. (2006). Precursors to college student burnout: Developing a typology of understanding. Qualitative Research Reports in Communication, 7(1), 23-31. https://doi.org/10.1080/17459430600964638

Desimone, L. (1999). Linking parent involvement with student achievement: Do race and income matter? Journal of Educational Research, 93(1), 11-30. https://doi.org/10.1080/00220679909597625

Dorrance Hall, E., \& Scharp, K. M. (2018). Testing a mediational model of the effect of family communication patterns on student perceptions of the impact of the college transition through social communication apprehension. Journal of Applied Communication Research, 46(4), 429-446. https://doi.org/10.1080/00909882.2018.1502461 
Dorrance Hall, E., McNallie, J., Custers, K., Timmermans, E., Wilson, S., \& VandenBulck, J. (2017). A cross-cultural examination of the mediating role of family support and parental advice quality on the relationship between family communication patterns and first-year college student adjustment in the United States and Belgium. Communication Research, 44, 638-667. https://doi.org/10.1177/0093650216657755

Faherty, A. N., Lowe, K., \& Arnett, J.J. (2020). Mind games: Parental psychological control and emerging adults' adjustment. Journal of Social and Personal Relationships, 37(2), 695714. https://doi.org/10.1177/0265407519877240

Furstenberg, F. F. (2015). Becoming adults: Challenges in the transition to adult roles. American Journal of Orothopsychiatry, 85(5), 14-21. https://doi.org/10.1037/ort0000107

Gridley, N., Bywater, T. J., \& Hutchings, J. M. (2018). Comparing live and video observation to assess early parent-child interactions in the home. Journal of Child \& Family Studies, 27(6), 1818-1829. https://doi.org/10.1007/s1082

Grolnick, W. S., Deci, E. L., \& Ryan, R. M. (1997). Internalization within the family: The selfdetermination theory perspective. In J. E. Grusec, \& L. Kuczynski (Eds.), Parenting and children's internalization of values: A handbook of contemporary theory (pp. 135-161). John Wiley.

Harackiewicz, J. M., Barron, K. E., Carter, S. M., Lehto, A. T., \& Elliot, A. J. (1997). Determinants and consequences of achievement goals in the college classroom: Maintaining interest and making the grade. Journal of Personality and Social Psychology, 73, 1284-1295. https://doi.org/10.1037/0022-3514.73.6.1284 
Hesse, C., Mikkelson, A. C., \& Saracco, S. (2018). Parent-child affection and helicopter parenting: Exploring the concept of excessive affection. Western Journal of Communication, 82(4), 457-474. https://doi.org/10.1080/10570314.2017.1362705

Higgins, E. T. (1987). Self-discrepancy: A theory relating self and affect. Psychological Review, 94, 319-340. https://doi.org/10.1037/0033-295X.94.3.319

Higgins, E. T., Strauman, T., \& Klein, R. (1986). Standards and the process of self-evaluation: Multiple affects from multiple stages. In R. M. Sorrentino \& E. T. Higgins (Eds.), Handbook of motivation and cognition: Foundations of social behavior (pp.23-63). The Guilford Press.

Holt, L. J., Mattanah, J. F., \& Long, M. W. (2018). Change in parental and peer relationship quality during emerging adulthood: Implications for academic, social, and emotional functioning. Journal of Social and Personal Relationships, 35(5), 743-769. https://doi.org/10.1177/0265407517697856

Infante, D. A., Myers, S. A., \& Buerkel, R. A. (1994). Argument and verbal aggression in constructive and deconstructive family and organizational disagreements. Western Journal of Communication, 58, 73-84. https://doi.org/10.1080/10570319409374488

Ingoglia, S., Inguglia, C., Liga, F., \& Lo Coco, A. (2016). Associations between perceived parental psychological control and internalizing difficulties in emerging adulthood: The role of identity. Journal of Social and Personal Relationships, 34(8), 1227-1240. https://doi.org/10.1177/0265407516670760 
Ishii, K., Mojaverian, T., Masuno, K., \& Kim, H. S. (2017). Cultural differences in motivation for seeking social support and the emotional consequences of receiving support: The role of influence and adjustment goals. Journal of Cross-Cultural Psychology, 48(9), 14421456. https://doi.org/10.1177/0022022117731091

Johnson, R. J. (2015). 10 Tensions in family discourse: Expectations and justifications. Linking Discourse Studies to Professional Practice. Multilingual Matters.

Johnson, R. J. (2018). The discursive practices of "guilting" in family discourse. Journal of Language Aggression \& Conflict, 6(2), 203-227. https://doi.org/10.1075/jlac.00010.joh

Johnson, D. I., \& Lewis, N. (2010, July-December). Perceptions of swearing in the work setting: An expectancy violations theory perspective. Communication Reports 23(2), 106-118. https://doi.org/10.1080.08934215.2010.511401

Jones, S., \& Bodie, G. (2014). Supportive communication. In Berger, C. R. (Ed.), Handbooks of Communication Science: Interpersonal Communication, 6, 371-394. Walter de Gruyter $\mathrm{GmbH}$.

Jose, P. E., \& Bellamy, M. A. (2012, September). Relationships of parents' theories of intelligence with children's persistence/learned helplessness: A cross-cultural comparison. Journal of Cross-Cultural Psychology, 43(6), 999-1018. https://doi.org/10.1177/0022022111421633

Kaniusonyte, G., \& Laursen, B. (2021). Parenting styles revisited: A longitudinal personoriented assessment of perceived parent behavior. Journal of Social and Personal Relationships, 38(1), 210-231._https://doi.org/10.1177/0265407520960818 
Karp, D. A., Holmstrom, L. L., \& Gray (1998). Leaving home for college: Expectations for selective reconstruction of self. Symbolic Interaction, 21(3), 253-276. https://doi.org/10.1525/si.1998.21.3.253

Knapp, M. L., Stohl, C., \& Reardon, K. (1981). 'Memorable' messages. Journal of Communication, 31(4), 27-41. https://doi.org/10.1111/j.1460-2466.1981.tb00448.x

Kriegbaum, K., Villarreal, B., Wu, V. V., \& Heckhausen, J. (2016). Parents still matter: Patterns of shared agency with parents predict college students' academic motivation and achievement. American Psychological Association, 2(2), 97-115. https://doi.org/doi.org/10.1037/mot0000033

Lanza-Kaduce, L., \& Webb, L. M. (1992, October). Perceived parental communication and adolescent self-esteem: Predictors of academic performance and drop-out rates. Association for Communication Administration Bulletin, 82, 1-12. https://commadministration.org/

Ledbetter, A. M. (2019). Parent-child privacy boundary conflict patterns during the first year of college: Mediating family communication patterns, predicting psychosocial distress. Human Communication Research, 45(3), 255-295. https://doi.org/10.1093/hcr/hqy018

Ledbetter, A. M., Beck, S. J. (2014). A theoretical comparison of relational maintenance and closeness as mediators of family communication patterns in parent-child relationships. Journal of Family Communication, 14(3), 230-252. https://doi.org/10.1080/15267431.2014.908196

Leung, C., Lau, S., \& Lam, W. (1998). Parenting styles and academic achievement: A crosscultural study. Merrill-Palmer Quarterly, 44(2), 157-172. https://www.jstor.org/stable/23093664 
Lindlof, T. R., \& Taylor, B. C. (2019). Qualitative communication research methods (4 ${ }^{\text {th }}$ ed). SAGE Publications, Inc.

Lynn, K., Duran, R. L., \& Miller-Ott, A. E. (2017). Helicopter parenting and cell-phone contact between parents and children in college. Southern Communication Journal, 82(2), 12https://doi.org/10.1080/1041794X.2017.1310286

Lynne, K., Miller-Ott, A. E., \& Duran, R. L. (2017, December). Sports scores and intimate moments: An expectancy violations theory approach to partner cell phone behaviors in adult romantic relationships. Western Journal of Communication, 81(5), 619-640. https://doi.org/10.1080/10570314.2017.1299206

MacGeorge, E. L., Samter, W., \& Gillihan, S. J. (2005). Academic stress, supportive communication, and health. Communication Education, 54(4), 365-372. https://doi.org/10.1080/03634520500442236

McKinney, C., \& Kwan, J. W. (2018). Emerging adult perceptions of and preferences for parenting styles and associated psychological outcomes. Journal of Family Issues, 39(9), 2491-2504. https://doi.org/10.1177/0192513X18756928

Michalos, A. C., Leung, C., \& Tsang Kit Man, S. (2014). Encyclopedia of quality of life and well-being research. Springer.

Miller-Ott, A. E. (2016). Helicopter parenting, family communication patterns, and out-of-class communication with college instructors. Communication Research Reports, 33(2), 173176. https://doi.org10.1080/08824096.2016.1154836

Miller, M., \& Day, L. E. (2002). Family communication, material and paternal expectations, and college students' suicidality. Journal of Family Communication, 2(4), 167-184. https://doi.org/10.1207/S15327698JFC0204_01 
Miller-Ott, A. E., \& Kelly, L. (2015). The presence of cell phones in romantic partner face-toface interactions: An expectancy violation theory approach. Southern Communication Journal, 80, 253-270. https://doi.org/10.1080/10510974.2015.1088876

Naumann, L. P., Guillaume, E. M., Funder. D. C. (2012). The correlates of high parental academic expectations: An Asian-Latino comparison. Journal of Cross-Cultural Psychology, 43(4), 515-520. https://doi.org/10.1177/0022022112438398

Nelson, L. J., Padilla-Walker, L. M., Carroll, J. S., Madsen, S. D., Barry, C. M., \& Badger, S. (2007). "If you want me to treat you like an adult, start acting like one!" Comparing the criteria that emerging adults and their parents have for adulthood. Journal of Family Psychology, 21, 665-674. https://doi.org/10.1037/0893-3200.21.4.665

Nelson, L. J., Padilla-Walker, L. M., Christnsen, K. J., Evans, C. A., \& Carroll, J. S. (2011). Parenting in emerging adulthood: An examination of parenting clusters and correlates. Journal of Youth Adolescence, 40, 730-743. https://doi.org/10.1007/s10964-010-9584-8

Nowell, L. S., Norris, J. M., White, D. E., \& Moules, N. J. (2017). Thematic analysis: Striving to meet the trustworthiness criteria. International Journal of Qualitative Methods, 16, 1-13. https://doi.org/10.1177/1609406917733847

Oishi, S., \& Sullivan, H. W. (2005). The mediating role of parental expectations in culture and well-being. Journal of Personality, 73, 1267-1294. https://doi.org/10.1111/j.14676494.2005.00349.x 
Padilla-Walker, L. M., \& Nelson, L. J. (2012). Black hawk down? Establishing helicopter parenting as a distinct construct from other forms of parental control during emerging adulthood. Journal of Adolescence, 35, 1177-1190. https://doi.org/10.1016/j.adolescence.2012.03.007

Phillipson, S., \& Phillipson, S. N. (2007). Academic expectations, beliefs of ability, and involvement by parents as predictors of child achievement: A cross-cultural comparison. Educational Psychology, 27(3), 329-348. https://doi.org/10.1080/01443410601104130

Planalp, S., Hafen, S., \& Adkins, A. D. (2000). Messages of shame and guilt. Annals of the International Communication Association, 23(1), 1-65. https://doi.org/10.1080/23808985.2000.11678969

Ritchie, L. D., \& Fitzpatrick, M. A. (1990). Family communication patterns: Measuring intrapersonal perceptions of interpersonal relationships. Communication Research, 523544. https://doi.org/10.1177/009365090017004007

Rittenour, C. E., Kromka, S. M., Saunders, R. K., Davis, K., Garlitz, K., Opatz, S. N., Sutherland, A., \& Thomas, M. (2019). Socializing the silent treatment: Parent and adult child communicated displeasure, identification, and satisfaction. Journal of Family Communication, 19(1), 77-93. https://doi.org/10.1080/15267431.2018.15431870

Ritzer, D. R., \& Sleigh, M. J. (2019). College students' comparative focus on grades, career preparation, and educated citizenry. Scholarship of Teaching and Learning in Psychology, 5(2), 148-161. https://doi.org/10.1037/st10000145

Rodriguez, P. M., Tan, L. X., \& Saleem, F. (2014). Shared burdens, personal costs on the emotional and social consequences of family honor. Journal of Cross-Cultural Psychology, 45(3), 400-416. https://doi.org/10.1177/0022022113511299 
Rostami, S., Ghanizadeh, A., \& Ghonsooly, B. (2015, September). External factors affecting second language motivation: The role of teacher burnout and family influence. Iranian Journal of Applied Linguistics, 18(2), 165-187. https://doi.org/10.18869/acadpub.ijal.18.2.165

Rudy, D., Carlo, G., Lamber, M. C., \& Awong, T. (2014). Undergraduates’ perceptions of parental relationship-oriented guilt induction versus harsh psychological control: Does cultural group status moderate their associations with self-esteem? Journal of CrossCultural Psychology, 45(6), 905-920. https://doi.org/10.1177/0022022114532354

Ryan, R. M., \& Deci, E. L. (2000). Self-determination theory and the facilitation of intrinsic motivation, social development, and well-being. American Psychologist, 55(1), 68-78. https://doi.org/10.1037110003-066X.55.1.68

Sansone, C., \& Smith, J. L. (2000). Interest and self-regulation: The relation between having to and wanting to. In C. Sansone, \& J. M. Harackiewicz (Eds.), Intrinsic and extrinsic motivation: The search for optimal motivation and performance (pp. 341-372). Academic Press.

Scheinfeld, E., \& Worley, T. (2018). Understanding the parent-child relationship during the transition into college and emerging adulthood using the relational turbulence theory. Communication Quarterly, 6(4), 444-462. https://doi.org/10.1080/01463373.2018.1443954

Schrodt, P., Witt, P. L., \& Messersmith, A. S. (2008). A meta-analytical review of family communication patterns and their association with information processing, behavioral, and psychosocial outcomes. Communication Monographs, 75(3), 248-269. https://doi.org/10.1080/03637750802256318 
Segrin, C., Woszidlo, A., Givertz, M., Bauer, A., \& Taylor Murphy, M. (2012). The association between overparenting, parent-child communication, and entitlement and adaptive traits in adult children. Family Relations, 61, 237-252. https://doi.org/10.1111/j.17413729.2011.00689.x

Silva, M., Dorso, E., Azhar, A., Renk, K. (2008). The relationship among parenting styles experienced during childhood, anxiety, motivation, and academic success in college students. Journal of College Student Retention, 9(2), 149-167. https://doi.org/10.2190/CS.9.2.b

Smetana, J. (1995). Parenting styles and conceptions of parental authority during adolescence. Child Development, 66, 299-316. https://doi.org/10.1111/j.1467-8624.1995.tb00872.x

Spitzberg, B. H., \& Hurt, H. T. (1987). The measurement of interpersonal skills in instructional contexts. Communication Education, 36, 28-45. https://doi.org/10.1080/03634528709378639

Spivey, L. A., Heubner, D. M., \& Diamond, L. A. (2018). Parent responses to childhood gender nonconformity: Effects of parent and child characteristics. Psychology of Sexual Orientation and Gender Diversity, 5(3), 360-370. https://doi.org/10.1037/sgd0000279

Stipek, D. Differences between Americans and Chinese in the cirucumstances evoking pride, shame, and guilt. (1998). Journal of Cross-Cultural Psychology, 29(5), 616-628. https://doi.org/10.1177/0022022198295002

Tanner, J. L., Arnett, J. J., Norcross, J. C., Freedheim, D. K., VandenBos, G. R., \& Domenech Rodriguez, M. M. (2016). Emerging adult clinical psychology. APA Handbook of Clinical Psychology: Roots and branches, 1, 127-138. https://doi.org/10.1037/14772-007 
Tang, J., Li, N., Sandoval, J., \& Liu, Y. (2018). Parenting styles and academic motivation: A sample from Chinese high schools. Journal of Child and Family Studies, 27, 33953401. https://doi.org/10.1007/s10826-018-1164-7

Tao, Y.K., \& Hong, Y. (2014). When academic achievement is an obligation: Perspectives from social-oriented achievement motivation. Journal of Cross-Cultural Psychology, 45(1), 110-136. https://doi.org/10.1177/0022022113490072

Vitoroulis, I., Schneider, B. H., Vasquez, C. C., de Toro, M. del P. S., \& Gonzales, Y. S. (2012, July). Perceived parental and peer support in relation to Canadian, Cuban, and Spanish adolescents' valuing of academics and intrinsic academic motivation. Journal of CrossCultural Psychology, 43(5), 704-722. https://doi.org/10.1177/0022022111405657

Wang, T. R. (2014, July-September). "I'm the only person from where I'm from to go to college:" Understanding the memorable messages first-generation college students receive from parents. Journal of Family Communication, 14(3), 270-290. https://doi.org/10.1080/15267431.2014.908195

Wang T. R., \& Nuru, A. (2017). "He wanted me to achieve that for our family and I did too": Exploring first-generation students' experiences of turning points during the transition to college. Journal of Family Communication, 17(2), 153-168. https://doi.org/10.1080/15267431.2016.1264401

Wang. L., \& Heppner, P. (2002). Assessing the impact of parental expectations and psychological distress on Taiwanese college students. The Counseling Psychologist, 30(4), 582-608. https://doi.org/10.1177/00100002030004006

Weidman, J. (1989). Undergraduate socialization: A conceptual approach. In J. Smart (Ed.), Higher education: Handbook of theory and research (Vol. 5, pp. 289-332). Agathon. 
Wintre, M. G., \& Yaffe, M. (2000). First-year students' adjustment to university life as a function of relationships with parents. Journal of Adolescent Research, 15(1), 9-37. https://doi.org/10.1177/0743558400151002

Wolfradt, U., Hempel, S., \& Miles, J. N. (2003). Perceived parenting styles, depersonalization, anxiety, and coping behavior in adolescents. Personality and Individual Differences, 34, 521-532. https://doi.org/10.1016/S0191-8869(02)00092-2

Youniss, J., \& Smollar, J. (1985). Adolescent relations with mothers, fathers, and friends. The University of Chicago Press. 


\section{APPENDIX A: RESEARCH BOARD STATEMENT}

If you recall (or still receive) messages from your parent(s) or primary caregiver(s) about college (e.g., success, achievement, majors, jobs, and/or priorities), and you think that these messages

have influenced your college experiences in some way, I'd love to speak with you! Please email me at akpate2@ilstu.edu 


\section{APPENDIX B: EMAIL RECRUITMENT}

Dear

I am a graduate student in the School of Communication and am conducting a research study to better understand how parental messages about college, success, achievement, majors, jobs, priorities, etc. may impact college students' experiences for my master's thesis. If you have or currently receive messages from your parents or primary caregivers that communicate messages about these entities for you in college, are comfortable speaking about the disclosures, and are at least 18 years old, then I request your participation.

You are invited to participate in an interview regarding the messages from your parent(s) or caregiver(s) that have impacted your perceptions of your college experiences. The interview may take place via phone, Facetime, Zoom, Skype, or in person (adhering to CDC, social distancing guidelines). Particularly, this study seeks to understand how messages students remember getting from parent(s) or caregiver(s) about college, success, achievement, etc. impact the college students' experiences.

The interview will take approximately 45-60 minutes. It will be confidential (I will not share your identity with others). I will change your name on the final paper for this project. Your participation will be completely voluntary. You may decide not to answer any question, and you can stop the interview at any time. All questions will focus on messages you remember receiving from your parent(s) or caregiver(s) and perceptions of your own college experiences, not on the relationship itself.

Please direct any questions and/or comments to my thesis advisor, Dr. Aimee Miller-Ott (aeott@ilstu.edu), or to me.

If you would like to participate, please contact me at the e-mail address below.

Sincerely, Anjali Patel

Graduate Student, School of Communication

Illinois State University

akpate2@ilstu.edu 


\section{APPENDIX C: FACEBOOK RECRUITMENT POST}

I am conducting a study for my thesis to better understand what messages college students remember receiving about college, success, achievement, majors, jobs, priorities, etc. I'm not asking people to speak about any relational or personal aspects of their relationship. Instead, I'm interested in what messages parent(s) or primary caregiver(s) have or still communicate to their children that impact how their collegiate student feels about their college experience. I am doing face-to-face, phone, or Skype/Facetime interviews that should last between 45 and 60 minutes. To participate, you must be able to recall past messages and/or ones you still receive about college from your parent(s) or caregiver(s) that you think may influence your college experiences. For example, "You need to go to college", "I expect you to keep in touch while at college", "All of your family members played football in college, you should do the same", If you ever need anything while you're away at school, you can always call me". You must be 18 years or older and feel comfortable speaking about this topic. Your responses will be confidential. I will not share your identity or anything you say to others in a way that will identify you. With your permission, I will audio record the interview and I will delete the recording once I transcribe the interview. I will also change your name on the final thesis. Anything you say answer will remain completely confidential. You can stop participating or skip questions any time you want. Please email me at akpate2@ilstu.edu if you want to participate. Please also send along this info to others and they can contact me directly to participate. 


\section{APPENDIX D: INFORMED CONSENT}

\section{Dear participants,}

I am a graduate student under the direction of Professor Aimee Miller-Ott in the School of Communication at Illinois State University. As part of the program requirement, I am conducting my thesis to better understand what messages students remember receiving from their parent(s) or primary caregiver(s) about college that have impacted their college experience.

Your participation will involve an open-ended interview about your communication with your parents. The interview will be conducted face-to-face, over phone, or through Facetime/Skype/Zoom and will take approximately 45 to 60 minutes. The interview will be audio recorded, with your permission, to ensure accuracy of recording your words. Recordings will be erased after transcription and will not have any links to your identity in the digital files. After the interview, you will receive an email from me with a questionnaire. You will be asked to complete and return the questionnaire to me via email. To participate, you must be able to recall (or still receive) messages about college from your parent(s) or caregiver(s) that have caused you to view your college experiences differently. You must be 18 years or older and feel comfortable speaking about the disclosure. The relationship must be a parent-child relationship. You are ineligible to participate if you are currently located in the European Economic Area.

Your participation in this study is voluntary. You may refuse to answer any question. You may skip or refuse to answer questions if you feel uncomfortable, and if you choose not to participate or to withdraw from the study at any time, there will be no penalty. Your responses are confidential - any information that might allow someone to identify you will not be disclosed. However, when required by law or university policy, identifying information (including your signed consent form) may be seen or copied by authorized individuals. After your data has been deidentified, your data may be used in other research projects. Your responses will be joined with those of other participants to develop themes for research presentation at conference or in publication.

As a participant, you may experience discomfort when talking about personal experiences. Participants may experience psychological or emotional pain. Participants who are distressed may contact Student Counseling Services at (309) 438-3655. Participants who are not ISU students may call the National Domestic Violence Hotline at 1-800-799-3224. Your comfort as a participant is of upmost importance. As a participant, you must feel comfortable speaking about your communication with your parents. If at any point you feel as though the interview is too uncomfortable, the interview will immediately end. You can also skip questions at any time. While your instructor may know who does or does not agree to participate in the research before grades are posted, you will not be penalized if you choose to not participate.

There is a risk of breach of confidentiality in this study. Participants may experience a risk to their reputation should a breach of confidentiality occur. To minimize this risk, participants are encouraged to choose private locations in which to answer interview questions. 
Please direct any questions and/or comments to Dr. Aimee Miller-Ott (aeott@ilstu.edu) or myself. If you have any questions about your rights as a subject/participant in this research, or if you feel you have been placed at risk, you can contact the Research Ethics \& Compliance Office at Illinois State University at (309) 438-5527.

Please respond to the following questions: 1) Do you agree to participate in this study? 2) Do you agree to be audio recorded?

You may print this form for your personal records.

Sincerely,

Anjali Patel, Graduate Student, School of Communication

Illinois State University

akpate2@ilstu.edu 


\section{APPENDIX E: INTERVIEW PROTOCOL}

Thank you for agreeing to participate in my study on parental expectations and college student experiences. I am going to ask you a series of open-ended questions regarding you and your parent(s) or caregiver(s) communication specifically focusing on moments where your parent(s) or caregiver(s) communicated expectations that influenced the ways in which you view your college experiences.

First, I'd like to ask you some background questions about yourself and your parent(s) or caregiver(s):

1. What parents or caregivers will you be reporting on? For example, your biological parents, your grandparents, stepparents, etc.

2. Did you live with your parent(s) or caregiver(s) before coming to college? If so, for how long before coming to school?

3. How often do you visit or speak with your parent(s) or caregiver(s) now that you are in college?

4. How long ago do you remember receiving messages from your parent(s) or caregiver(s) about college?

5. What was your age at the time messages began?

6. Do your parent(s) or caregiver(s) have college-level degrees?

7. What are your personal future goals?

Next, I'd like to talk about your conversational relationship with your parent(s) or caregiver(s):

8. How would you describe the types of conversations you and your parent(s) or caregiver(s) have? For example, do you tend to have open dialogue about decisions, not just ones focused on college?

9. How would you describe how you make your own decisions in college? For example, do you make a lot of decisions on your own, do you talk to your friends or parents or caregivers first, does it matter what you are making decisions about? If so, how does it matter?

a. How do you respond to your parent(s) or caregiver(s) decision making advice? For example, do you always accept their advice? Do you ever challenge their advice or voice your own ideas?

b. How often, if at all, do your parent(s) or caregiver(s) offer decision making guidance? Do you always have to request guidance? Or, do they offer their advice without your having asked for it?

c. How do your parent(s) or caregiver(s) respond if you don't follow their decision making suggestions?

d. Do you feel comfortable and confident making your own decisions in college?

10. How involved would you say your parent(s) or caregiver(s) are in your college education/experiences? For example, do you communicate with your parents about school, daily? Do you rarely communicate with your parents about school?

a. Follow up: Do you ever feel obligated to share your academic success with your parents? 
b. Follow up: Do you ever feel obligated to communicate with your parents on a regular basis?

c. How often do you share your own college education/experiences with your parents?

d. How often do your parents ask you about your college education/experiences? For example, do your parents often ask how you are doing in college (e.g. academically, socially)?

e. Would you describe your parent(s) or caregiver(s) as overinvolved with your college education/experiences?

f. Do you ever feel that your parent(s) or caregiver(s) overstep boundaries in your relationship?

11. In what ways have your parents or caregivers helped you to become independent in your life and now that you are in college?

a. If not, how, if at all, have your parents or caregivers made you feel dependent in life now that you are in college?

\section{Next, I'm going to ask you some messages you might recall from your parent(s) or caregiver(s):}

12. What messages can you remember getting from your parent(s) or caregiver(s) in the past or more recently about college, majors or jobs, success, and priorities? For example, have they ever shared messages about which major you might select, or what extra-curricular opportunities you might enroll in?

13. How have conversations about college taken place? For example, did they ever sit you down and talk about college? Are they more casual conversations? Do they happen on the phone or FaceTime? When did these messages or communication instances typically take place? Such as, were you alone? Were you around other people or family?

14. Can you explain to me, in the timeline of the relationship, when or how often your parent(s) or caregiver(s) shared these messages with you? Can you recall how you responded? What do you typically do with these messages? For example, do you do what they suggest? Do you argue back? Do you ignore their messages?

a. Follow up: How do your parent(s) or caregiver(s) handle your responses to their messages?

15. How, if at all, do you see these messages conveying what your parent(s) or caregiver(s) expect of you?

a. Do you think your culture has impacted the types of messages your parent(s) or caregiver(s) elicit?

Now I'd like to focus on your experiences with these messages. You will be asked to recall specific conversations with your parent(s) or caregiver(s), all within your comfort zone. Please note that I am asking for recalled, verbal messages only.

16. What do you think your parent(s) or caregiver(s) were hoping to accomplish with these messages?

a. How have these messages your parent(s) or caregiver(s) shared with you made you feel? 
17. Looking back now or at the time, did (do) you ever consider these messages as impactful on your mental health and overall well-being? For example, have you ever felt stressed or sad as a result of these messages?

a. Follow up: What made you realize these messages were causing the impact?

18. How, if at all, would you say these messages or expectations have impacted your college experiences in the classroom and with classes/professors and also in other areas like socially with friends?

a. In your experiences with these messages, have you ever felt lonely or obligated in any way?

b. Would you say that you have ever sacrificed social opportunities as a result of these messages?

c. Would you say that these messages have ever caused others to ridicule you?

d. Have your parent(s) or caregiver(s) ever expressed anger or frustration at the thought of you going out?

19. Would you say that these messages have impacted your academic achievement or success? For example, do you ever worry about earning satisfactory grades?

a. How, if at all, have these messages helped you feel or become successful and achieving? Or, how have they not?

e. Follow up: How, if at all, have these messages impacted your motivation or persistence?

f. Do you feel that these messages cause you to set unrealistic or unreal goals for yourself?

20. How did you view your parent(s) or caregiver(s) when they used these messages? For example, did you perceive them to be controlling, well-wishing, overbearing?

g. Follow up: How, if at all, do you think the ways your parent(s) or caregiver(s) raised you is reflected within the messages they share with you?

21. How, if at all, have these messages from your parents or caregivers changed how you communicate with peers? How have these messages changed how you communicate with your instructors? For example, do you tend to avoid office hours, do you work hard to communicate your desire to do well?

h. Follow up: How do you feel about how these messages have impacted these relationships?

22. Have these messages changed how you react and respond to messages of criticism? If so, how? For example, do you tend to take instructor feedback personally?

23. In what other ways do you think messages from your parent(s) or caregiver(s) about college have affected you and your college experiences and choices?

What suggestions would you have for someone who has received, or still receives, messages from their parents or caregivers about what they suggest or expect of them in college?

Is there anything else you would like to share concerning messages you recall receiving from your parent(s) or caregiver(s) about college? 